\title{
T-CONVERGENCE FOR FUNCTIONALS DEPENDING ON VECTOR FIELDS. I. INTEGRAL REPRESENTATION AND COMPACTNESS.
}

\author{
A. MAIONE, A. PINAMONTI, AND F. SERRA CASSANO \\ Abstract. Given a family of locally Lipschitz vector fields $X(x)=$ \\ $\left(X_{1}(x), \ldots, X_{m}(x)\right)$ on $\mathbb{R}^{n}, m \leq n$, we study functionals depending on \\ $X$. We prove an integral representation for local functionals with respect \\ to $X$ and a result of $\Gamma$-compactness for a class of integral functionals \\ depending on $X$.
}

\section{INTRODUCTION}

In this paper we will deal with the $\Gamma$-convergence, with respect to $L^{p}(\Omega)$ topology, for integral functionals $F, F_{1}: L^{p}(\Omega) \rightarrow[0, \infty], 1<p<\infty$, defined by

$$
F(u):= \begin{cases}\int_{\Omega} f(x, X u(x)) d x & \text { if } u \in \mathbf{C}^{1}(\Omega) \\ \infty & \text { otherwise }\end{cases}
$$

and

$$
F_{1}(u):=\left\{\begin{array}{ll}
\int_{\Omega} f(x, X u(x)) d x & \text { if } u \in W_{\text {loc }}^{1,1}(\Omega) \\
\infty & \text { otherwise }
\end{array},\right.
$$

where $X(x):=\left(X_{1}(x), \ldots, X_{m}(x)\right)$ is a given family of first linear differential operators, with Lipschitz coefficients on a bounded open set $\Omega \subset \mathbb{R}^{n}$, that is,

$$
X_{j}(x)=\sum_{i=1}^{n} c_{j i}(x) \partial_{i} \quad j=1, \ldots, m
$$

with $c_{j i}(x) \in \operatorname{Lip}(\Omega)$ for $j=1, \ldots, m, i=1, \ldots, n$ and where $f: \Omega \times \mathbb{R}^{m} \rightarrow$ $[0, \infty]$ is a Borel function. In the following, we will refer to $X$ and $f$ as $X$-gradient and integrand function, respectively. As usual, we will identify

Date: April 16, 2019.

A.M is supported by MIUR, Italy, GNAMPA of INDAM and University of Trento, Italy.

A.P. is supported by MIUR, Italy, GNAMPA of INDAM and University of Trento, Italy.

F.S.C. is supported by MIUR, Italy, GNAMPA of INDAM and University of Trento, Italy. 
each $X_{j}$ with the vector field $\left(c_{j 1}(x), \ldots, c_{j n}(x)\right) \in \operatorname{Lip}\left(\Omega, \mathbb{R}^{n}\right)$. Moreover, we set

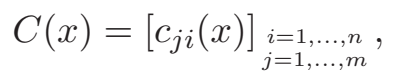

and we will call $C(x)$ the coefficient matrix of the $X$-gradient.

Throughtout the paper the class of integrand functions will tipically satisfy the following structural conditions:

$\left(I_{1}\right)$ for every $\eta \in \mathbb{R}^{m}$, the function $f(\cdot, \eta): \Omega \rightarrow[0, \infty]$ is Borel measurable on $\Omega$;

$\left(I_{2}\right)$ for a.e. $x \in \Omega$, the function $f(x, \cdot): \mathbb{R}^{m} \rightarrow[0, \infty)$ is convex;

$\left(I_{3}\right)$ there exists constants $c_{1}>c_{0} \geq 0$ such that

$$
c_{0}|\eta|^{p} \leq f(x, \eta) \leq c_{1}\left(|\eta|^{p}+1\right),
$$

for a.e. $x \in \Omega$ and for each $\eta \in \mathbb{R}^{m}$.

We will denote by $I_{m, p}\left(\Omega, c_{0}, c_{1}\right)$ the class of such integrand functions. Notice that both functionals (11) and (2) always admit an integral representation with respect to the Euclidean gradient. Indeed, for instance, functional (1) can be represented as follows

$$
F(u)=\int_{\Omega} f_{e}(x, D u) d x \text { for each } u \in \mathbf{C}^{1}(\Omega)
$$

where $f_{e}: \Omega \times \mathbb{R}^{n} \rightarrow[0, \infty]$ now denotes the Euclidean integrand defined as

$$
f_{e}(x, \xi):=f(x, C(x) \xi) \quad \text { for a.e. } x \in \Omega \text {, for each } \xi \in \mathbb{R}^{n} .
$$

Notice also that, in general, we cannot reverse this representation (see Counterexample 3.15). Moreover the representation with respect to the Euclidean gradient could yield a loss of coercivity. Indeed, for instance, let us consider as $X$-gradient the Grushin and Heisenberg vector fields in Example 2.2 (ii) and (iii), respectively, and let $f(x, \eta)=|\eta|^{2}$. Then, it is easy to see that there are no positive constants $c>0$ such that the associated Euclidean integrand $f_{e}(x, \xi)=f(x, C(x) \xi)=|C(x) \xi|^{2}$ satisfies

$$
f_{e}(x, \xi) \geq c|\xi|^{2} \text { for a.e. } x \in \Omega, \forall \xi \in \mathbb{R}^{n},
$$

if the open set $\Omega \subset \mathbb{R}^{2}$ contains some segment of the line $\left\{x_{1}=0\right\}$, for the Grushin vector field, and for each open set $\Omega \subset \mathbb{R}^{3}$, for the Heisenberg vector fields. Nonetheless, we will show that, by replacing the Euclidean gradient with the $X$-gradient, we can get rid of this drawback.

Functional (11) was studied in [FSSC1] as far as its relaxation and in connection with the so-called Meyers-Serrin theorem for Sobolev spaces associated with the $X$-gradient, denoted $W_{X}^{1, p}(\Omega)$ (see Definition 2.3 and [FS]). As a consequence, the following characterization of relaxed functionals $\bar{F}$ and $\bar{F}_{1}$ can be given (see (22) and Theorem 3.1): if $f \in I_{m, p}\left(\Omega, c_{0}, c_{1}\right)$ with $c_{1} \geq c_{0}>0$ and $F^{*}: L^{p}(\Omega) \rightarrow[0, \infty]$ denotes the functional

$$
F^{*}(u):=\left\{\begin{array}{ll}
\int_{\Omega} f(x, X u(x)) d x & \text { if } u \in W_{X}^{1, p}(\Omega) \\
\infty & \text { otherwise }
\end{array},\right.
$$


then

$$
\bar{F}(u)=\bar{F}_{1}(u)=F^{*}(u) \quad \forall u \in L^{p}(\Omega) .
$$

By (6) and a well-known property of $\Gamma$-convergence (see [DM, Propostion 6.11]), the characterization of $\Gamma$-limits for functionals of type (11) or (2), associated to integrand functions in $I_{m, p}\left(\Omega, c_{0}, c_{1}\right)$, can be reduced to the one for functionals of type (5) still associated to integrand functions in $I_{m, p}\left(\Omega, c_{0}, c_{1}\right)$. For getting such a characterization, the following structure assumption on the $X$-gradient turns out to be a key point.

1.1. Definition. We say that the family of vector fields $X(x)=\left(X_{1}(x)\right.$, $\left.\ldots, X_{m}(x)\right)$ on an open set $\Omega \subset \mathbb{R}^{n}$ satisfies the linear independence condition (LIC) if there exists a closed set $\mathcal{N}_{X} \subset \Omega$ such that $\left|\mathcal{N}_{X}\right|=0$ and, for each $x \in \Omega_{X}:=\Omega \backslash \mathcal{N}_{X}, X_{1}(x), \ldots, X_{m}(x)$ are linearly independent as vectors of $\mathbb{R}^{n}$.

Let us point out that (LIC) condition embraces many relevant families of vector fields studied in literature (see Example 2.2). In particular neither the Hörmander condition for $X$, that is, vector fields $X_{j}$ 's are smooth and the rank of the Lie algebra generated by $X_{1}, \ldots, X_{m}$ equals $n$ at any point of $\Omega$, nor the (weaker) assumption that the $X$-gradient induces a CarnotCarathéodory metric in $\Omega$ is requested. An exaustive account of these topics can be found in BLU].

The main results of this paper are the following (see Theorems 3.12 and 4.11).

- Assume that the $X$-gradient satisfies (LIC) on $\Omega$ and let us denote by $\mathcal{A}$ the class of open sets contained in $\Omega$. Then an integral representation result, with respect to the $X$-gradient, is provided for a local functional $F: L^{p}(\Omega) \times \mathcal{A} \rightarrow[0, \infty]$ satisfying suitable assumptions.

- Assume that the $X$-gradient satisfies (LIC) on $\Omega$, and let $F_{h}^{*}$ : $L^{p}(\Omega) \rightarrow[0, \infty](h=1,2, \ldots)$ be a sequence of integral functionals of the form (5) with $f \equiv f_{h}$, where $\left(f_{h}\right)_{h} \subset I_{m, p}\left(\Omega, c_{0}, c_{1}\right)$ for given constants $0<c_{0} \leq c_{1}$. Then, up to a subsequence, $\left(F_{h}^{*}\right)_{h} \Gamma$ converges, in $L^{p}(\Omega)$-topology, to a functional $F^{*}: L^{p}(\Omega) \rightarrow[0, \infty]$, and $F^{*}$ can be still represented as in (5), for a suitable integrand function $f \in I_{m, p}\left(\Omega, c_{0}, c_{1}\right)$.

We will also single out two signifiant integrand function subclasses $J_{i} \subset$ $I_{m, p}\left(\Omega, c_{0}, c_{1}\right)(i=1,2)$ for which the associated functionals in (5) are still compact with respect to $\Gamma$ - convergence with respect to $L^{p}(\Omega)$-topology (see Theorem 4.20).

The techniques for showing the integral representation Theorem 3.12 rely on the analogous classical integral representation result for the Euclidean gradient (see [DM, Theorem 20.1] ), together with a characterization of integral functionals depending on the Euclidean gradient which can be also represented with respect to a given $X$-gradient (see Theorem 3.5). Let us stress that we cannot here exploit, as in the case of the Euclidean gradient, 
the approximation by piecewise-affine functions in classical Sobolev space $W^{1, p}(\Omega)$, since it could not work in Sobolev space $W_{X}^{1, p}(\Omega)$ (see section 2.3). The strategy for showing the $\Gamma$-compactness Theorem 4.11 will consists of two steps.

1st step. By applying classical results contained in [DM], we will prove the following result (see Theorem 4.18): let $\left(f_{h}\right)_{h} \subset I_{m, p}\left(\Omega, c_{0}, c_{1}\right)$, let $\left(F_{h}\right)_{h}$ be a sequence of integral functionals on $L^{p}(\Omega) \times \mathcal{A}, 1<p<\infty$, of the form

$$
F_{h}(u, A):=\left\{\begin{array}{ll}
\int_{A} f_{h, e}(x, D u(x)) d x & \text { if } A \in \mathcal{A}, u \in W_{\mathrm{loc}}^{1,1}(A) \\
\infty & \text { otherwise }
\end{array},\right.
$$

where

$$
f_{h, e}(x, \xi):=f_{h}(x, C(x) \xi) \quad x \in \Omega, \xi \in \mathbb{R}^{n} .
$$

Then, up to a subsequence, there exists $F: L^{p}(\Omega) \times \mathcal{A} \rightarrow[0, \infty]$ such that

$$
F(\cdot, A)=\Gamma\left(L^{p}(\Omega)\right)-\lim _{h \rightarrow \infty} F_{h}(\cdot, A) \quad \text { for each } A \in \mathcal{A},
$$

and $F$ can be represented by an integral form on $W^{1, p}(A)$ by means of an Euclidean integrand function, that is,

$$
F(u, A):=\int_{A} f_{e}(x, D u(x)) d x
$$

for every $A \in \mathcal{A}$, for every $u \in L^{p}(\Omega)$ such that $\left.u\right|_{A} \in W^{1, p}(A)$ for a suitable Borel function $f_{e}: \Omega \times \mathbb{R}^{n} \rightarrow[0, \infty]$.

2nd step. We will show that the class $I_{m, p}\left(\Omega, c_{0}, c_{1}\right)$ satisfies the following closure property with respect to $\Gamma\left(L^{p}(\Omega)\right.$ )-convergence (see Theorem 4.19): assume that $\left(f_{h}\right)_{h} \subset I_{m, p}\left(\Omega, c_{0}, c_{1}\right)$ and (9) and (10) hold, then $F$ satisfies the assumptions of the integral representation Theorem 3.12. Thus $F$ can be also represented in the integral form (5), by means of an integrand function $f \in I_{m, p}\left(\Omega, c_{0}, c_{1}\right)$.

Eventually let us point out that the $\Gamma$-convergence for functionals such as in (11) have been studied in the framework of Dirichlet forms [MR, Fu], but for special integrand functions $f$ and $X$-gradient satisfying the Hörmander condition,(see, for instance, $[\mathrm{MO}, \overline{\mathrm{BT}}]$ and references there in). Other variational convergences, such as homogenization and $H$-convergence for subelliptic PDEs have been also widely studied, always assuming the $X$-gradient satisfying the Hörmander condition (see, for instance, BMT, BPT1, BPT2, FT, FGVN, FTT, BFT, BFTT and the references there in). In the subsequent paper [MPSC] we will be concerned with relationships between $\Gamma$ convergence of functionals depending on vector fields and convergence of their minimizers. Thus, we will refer to MPSC] for a comparison among our results with those already present in literature.

Acknowledgements. We thank A. Braides, G. Buttazzo, G. Dal Maso, A. Defranceschi and B. Franchi for useful suggestions and discussions on this topic. 


\section{Vector fields and Sobolev spaces Depending on Vector fieldS}

2.1. Notation and definitions. Through this paper $\Omega \subset \mathbb{R}^{n}$ is a fixed open set and $\overline{\mathbb{R}}=[-\infty, \infty]$. If $v, w \in \mathbb{R}^{n}$, we denote by $|v|$ and $\langle v, w\rangle$ the Euclidean norm and the scalar product, respectively. If $\Omega$ and $\Omega^{\prime}$ are subsets of $\mathbb{R}^{n}$ then $\Omega^{\prime} \Subset \Omega$ means that $\Omega^{\prime}$ is compactly contained in $\Omega$. Moreover, $B(x, r)$ is the open Euclidean ball of radius $r$ centered at $x$. Sometimes we will denote by $B^{k}(x, r)$ the open Euclidean ball of radius $r$ centered at $x \in \mathbb{R}^{k}$ in $\mathbb{R}^{k}$. If $A \subset \mathbb{R}^{n}$ then $\chi_{A}$ is the characteristic function of $A,|A|$ is its n-dimensional Lebesgue measure $\mathcal{L}^{n}$ and by notation a.e. $x \in A$, we will simply mean $\mathcal{L}^{n}$-a.e. $x \in A$.

In the sequel we denote by $\mathbf{C}^{k}(\Omega)$ the space of $\mathbb{R}$-valued functions $k$ times continuously differentiable and by $\mathbf{C}_{c}^{k}(\Omega)$ the subspace of $\mathbf{C}^{k}(\Omega)$ whose functions have support compactly contained in $\Omega$.

We will use spherically symmetric mollifiers $\rho_{\epsilon}$ defined by $\rho_{\epsilon}(x):=\epsilon^{-n} \rho\left(\epsilon^{-1}|x|\right)$, where $\rho \in \mathbf{C}_{c}^{\infty}([-1,1]), \rho \geq 0$ and $\int_{0}^{1} \rho(t) d t=|B(0,1)|^{-1}$.

For any $u \in L^{1}(\Omega)$ define $X u$ as an element of $\mathcal{D}^{\prime}\left(\Omega ; \mathbb{R}^{m}\right)$ as follows

$$
\begin{aligned}
& X u(\psi):=\left(X_{1} u\left(\psi_{1}\right), \ldots, X_{m} u\left(\psi_{m}\right)\right) \\
& =-\int_{\Omega} u\left(\sum_{i=1}^{n} \partial_{x_{i}}\left(c_{1, i} \psi_{1}\right), \ldots, \sum_{i=1}^{n} \partial_{x_{i}}\left(c_{m, i} \psi_{m}\right)\right) d x \\
& \forall \psi=\left(\psi_{1}, \ldots, \psi_{m}\right) \in \mathbf{C}_{c}^{\infty}\left(\Omega ; \mathbb{R}^{m}\right) \text {. If we set } X^{T} \psi:=\left(X_{1}^{T} \psi_{1}, \ldots, X_{m}^{T} \psi_{m}\right) \\
& \text { with } \\
& X_{j}^{T} \varphi:=\int_{\Omega} \sum_{i=1}^{n} \partial_{x_{i}}\left(c_{j, i} \varphi\right) d x \quad \forall \varphi \in \mathbf{C}_{c}^{\infty}(\Omega), \forall j=1, \ldots, m,
\end{aligned}
$$

the aspect of the definition is even more familiar

$$
X u(\psi):=-\int_{\Omega} u X^{T} \psi d x \quad \forall \psi \in \mathbf{C}_{c}^{\infty}\left(\Omega ; \mathbb{R}^{m}\right) .
$$

2.1. Remark. If $X=\left(X_{1}, \ldots, X_{m}\right)$ satisfies (LIC) on an open set $\Omega \subset \mathbb{R}^{n}$, then $m \leq n$. Moreover, by the well-known extension result for Lipschitz functions, without loss of generality, we can assume that vector fields' coefficients $c_{j i} \in \operatorname{Lip}$ loc $\left(\mathbb{R}^{n}\right)$ for each $j=1, \ldots, m, i=1, \ldots, n$.

2.2. Example (Relevant vector fields).

(i) (Euclidean gradient ) Let $X=\left(X_{1}, \ldots, X_{n}\right)=D:=\left(\partial_{1}, \ldots, \partial_{n}\right)$. In this case the coefficients matrix $C(x)$ of $X$ is a $n \times n$ matrix and

$$
C(x)=\mathrm{I}_{\mathrm{n}} \quad \forall x \in \mathbb{R}^{n},
$$

denoting $I_{n}$ the identity matrix of order $n$.

(ii) (Grushin vector fields) Let $X=\left(X_{1}, X_{2}\right)$ be the vector fields on $\mathbb{R}^{2}$ defined as

$$
X_{1}(x):=\partial_{1}, \quad X_{2}(x):=x_{5} \partial_{2} \text { if } x=\left(x_{1}, x_{2}\right) \in \mathbb{R}^{2} .
$$


In this case the coefficients matrix $C(x)$ of $X$ is a $2 \times 2$ matrix and

$$
C(x):=\left[\begin{array}{cc}
1 & 0 \\
0 & x_{1}
\end{array}\right]
$$

(iii) (Heisenberg vector fields) Let $X=\left(X_{1}, X_{2}\right)$ be the vector fields on $\mathbb{R}^{3}$ defined as

$$
X_{1}(x):=\partial_{1}-\frac{x_{2}}{2} \partial_{3}, X_{2}(x):=\partial_{2}+\frac{x_{1}}{2} \partial_{3} \text { if } x=\left(x_{1}, x_{2}, x_{3}\right) \in \mathbb{R}^{3} .
$$

In this case the coefficients matrix $C(x)$ of $X$ is a $2 \times 3$ matrix and

$$
C(x):=\left[\begin{array}{ccc}
1 & 0 & -x_{2} / 2 \\
0 & 1 & x_{1} / 2
\end{array}\right]
$$

Notice that all three families of vector fields satisfy (LIC) respectively in $\Omega=\mathbb{R}^{n}, \Omega=\mathbb{R}^{2}$ and $\Omega=\mathbb{R}^{3}$. Indeed, it suffices to take $\Omega_{X}=\Omega$ in $(i)$ and $\left(\right.$ ii) and $\Omega_{X}=\Omega \backslash \mathcal{N}_{X}$ with $\mathcal{N}_{X}:=\left\{\left(0, x_{2}\right): x_{2} \in \mathbb{R}\right\}$ in (ii). Moreover they are locally Lipschitz continuous in $\Omega$.

2.3. Definition. For $1 \leq p \leq \infty$ we set

$$
\begin{aligned}
W_{X}^{1, p}(\Omega) & :=\left\{u \in L^{p}(\Omega): X_{j} u \in L^{p}(\Omega) \text { for } j=1, \ldots, m\right\} \\
W_{X ; l o c}^{1, p}(\Omega) & :=\left\{u:\left.u\right|_{\Omega^{\prime}} \in W_{X}^{1, p}\left(\Omega^{\prime}\right) \text { for every open set } \Omega^{\prime} \Subset \Omega\right\}
\end{aligned}
$$

2.4. Remark. Since vector fields $X_{j}$ have locally Lipschitz continuous coefficients, $\partial_{i} c_{j, i} \in L_{\text {loc }}^{\infty}\left(\mathbb{R}^{n}\right)$ for each $j=1, \ldots, m, i=1, \ldots, n$, thus, by definition, it is immediate that, for each open bounded set $\Omega \subset \mathbb{R}^{n}$,

$$
W^{1, p}(\Omega) \subset W_{X}^{1, p}(\Omega) \quad \forall p \in[1, \infty],
$$

and for any $u \in W^{1, p}(\Omega)$

$$
X u(x)=C(x) D u(x) \quad \text { for a.e. } x \in \Omega,
$$

where $W^{1, p}(\Omega)$ denotes the classical Sobolev space, or, equivalently, the space $W_{X}^{1, p}(\Omega)$ associated to $X=D:=\left(\partial_{x_{1}}, \ldots, \partial_{x_{n}}\right.$ ) (see Example 2.2 (i)). Moreover it is easy to see that inclusion (14) can be strict and turns out to be continuous. As well, there is the inclusion

$$
W_{\text {loc }}^{1, p}(\Omega) \subset W_{X ; \mathrm{loc}}^{1, p}(\Omega) \quad \forall p \in[1, \infty],
$$

The following Proposition is proved in [FS]

2.5. Proposition. $W_{X}^{1, p}(\Omega)$ endowed with the norm

$$
\|u\|_{W_{X}^{1, p}(\Omega)}:=\|u\|_{L^{p}(\Omega)}+\sum_{i=1}^{m}\left\|X_{j} u\right\|_{L^{p}(\Omega)}
$$

is a Banach space, reflexive if $1<p<\infty$.

2.6. Remark. The following properties hold for functions in $W_{X ; \text { loc }}^{1, p}(\Omega)$ : 
(i) let $u \in L^{p}(\Omega)$ and assume there exists an open set $A \subset \Omega$ such that $\left.u\right|_{A} \in W_{X ; \text { loc }}^{1, p}(A)$. Then, for every open set $A^{\prime} \Subset A$, there exists

$$
w \in W_{X}^{1, p}(\Omega) \text { such that }\left.u\right|_{A^{\prime}}=\left.w\right|_{A^{\prime}} .
$$

Indeed, there exists a cut-off function $\varphi \in \mathbf{C}_{c}^{1}(A)$ such that $\varphi \equiv 1$ in $A^{\prime}$. If

$$
w(x):=u(x) \varphi(x) \text { if } x \in \Omega,
$$

then it is easy to see that $w$ satisfies (17).

(ii) Let $\left\{A_{1}, \ldots, A_{N}\right\}$ be a finite family of open subsets of $\Omega$ and let $u \in L^{p}(\Omega)$. If $u_{\mid A_{i}} \in W_{X}^{1, p}\left(A_{i}\right)$ for all $i=1, \ldots, N$ then $u \in$ $W_{X}^{1, p}\left(\bigcup_{i=1}^{N} A_{i}\right)$. Consider a partition of unity subordinate to the covering $\left\{A_{1}, \ldots, A_{N}\right\}$, i.e., nonnegative functions $\left\{\eta_{1}, \ldots, \eta_{N}\right\} \subset$ $C_{c}^{\infty}\left(\bigcup_{i=1}^{N} A_{i}\right)$ such that each $\eta_{j}$ has support in some $A_{i}$ and $\sum_{j=1}^{N} \eta_{j}(x)=$ 1 for all $x \in \bigcup_{i=1}^{N} A_{i}$. Set $u_{j}=u \eta_{j}$. Since the support of $\eta_{j}$ is contained in some $A_{i}$, it is clear that $u_{j} \in W_{X}^{1, p}\left(\bigcup_{i=1}^{N} A_{i}\right)$. The conclusion follows observing that $u=\sum_{j=1}^{N} u_{j}$.

(iii) Let $A \subset \Omega$ be an open subset and let $u \in L^{p}(A)$ be such that there exists $M>0,\|u\|_{W_{X}^{1, p}\left(A^{\prime}\right)} \leq M$ for any $A^{\prime} \Subset A$, then $u \in W_{X}^{1, p}(A)$. It is easy to see that $u$ admits the weak gradient $X u$. Consider a sequence of open subsets of $A,\left\{A_{i}\right\}_{i \in \mathbb{N}}$ with $A_{i} \Subset A_{i+1}$ and $A \subseteq$ $\bigcup_{i=1}^{\infty} A_{i}$

$$
\int_{A}|X u|^{p} d x \leq \int_{\bigcup_{i=1}^{\infty} A_{i}}|X u|^{p} d x=\lim _{i \rightarrow \infty} \int_{A_{i}}|X u|^{p} d x \leq M
$$

and the conclusion follows.

(iv) Let $A \subset \Omega$ be an open subset and $u \in W_{X}^{1, p}(A)$, then $u_{\mid B} \in W_{X}^{1, p}(B)$ for any open set $B \subseteq A$. The thesis follows easily observing that $C_{c}^{\infty}(B) \subseteq C_{c}^{\infty}(A)$.

2.2. Approximation by regular functions. Let us recall in this section some results of approximation by regular functions in these anisotropic Sobolev spaces. In particular the analogous of the celebrated Meyers-Serrin theorem, proved, independently, in [FSSC1 and [GN]. Analogous results (under some additional assumptions) in the weighted cases are proved in [FSSC2], see also [APS] for a generalization to metric measure spaces.

Here and in the sequel, if $u: \Omega \rightarrow \overline{\mathbb{R}}$, we will denote by $\bar{u}: \mathbb{R}^{n} \rightarrow \overline{\mathbb{R}}$ its extension to the whole $\mathbb{R}^{n}$ being 0 outside of $\Omega$.

2.7. Proposition. Assume $u \in W_{X}^{1, p}(\Omega)$ for $1 \leq p<\infty$. Then if $\Omega^{\prime} \Subset \Omega$

$$
\lim _{\epsilon \rightarrow 0}\left\|\bar{u} * \rho_{\epsilon}-u\right\|_{W_{X}^{1, p}\left(\Omega^{\prime}\right)}=0,
$$

where $\rho_{\epsilon}(x)=\epsilon^{-n} \rho\left(\epsilon^{-1}|x|\right)$ is a mollifier supported in $B(0, \epsilon)$. 
2.8. Definition. For $1 \leq p \leq \infty$ we set

$$
H_{X}^{1, p}(\Omega):=\text { closure of } \mathbf{C}^{1}(\Omega) \cap W_{X}^{1, p}(\Omega) \text { in } W_{X}^{1, p}(\Omega)
$$

As for the usual Sobolev spaces $H_{X}^{1, p}(\Omega) \subset W_{X}^{1, p}(\Omega)$. The classical result ' $H=W$ 'of Meyers and Serrin ([MS] still holds for these anisotropic Sobolev spaces.

2.9. Theorem. Let $\Omega$ be an open subset of $\mathbb{R}^{n}$ and $1 \leq p<\infty$. Then

$$
H_{X}^{1, p}(\Omega)=W_{X}^{1, p}(\Omega) .
$$

The proofs of Proposition 2.7 and Theorem 2.9 can be found in [FSSC1] and $\mathrm{GN}$.

Let us collect below some well-known properties about approximation by convolution and convex functions.

2.10. Proposition. (i) Let $\left(u_{h}\right)_{h}$ and $u$ be in $L_{l o c}^{p}\left(\mathbb{R}^{n}\right)$ and let $\Omega \subset \mathbb{R}^{n}$ be a bounded open set such that

$$
u_{h} \rightarrow u \text { in } L_{l o c}^{1}(\Omega) \text { as } h \rightarrow \infty .
$$

Then, for each open set $\Omega^{\prime} \Subset \Omega$, for given $0<\epsilon<\operatorname{dist}\left(\Omega^{\prime}, \mathbb{R}^{n} \backslash \Omega\right)$,

$$
\rho_{\epsilon} * u_{h} \rightarrow \rho_{\epsilon} * u \text { uniformly on } \Omega^{\prime} \text {, as } h \rightarrow \infty \text {. }
$$

(ii) Let $f: \mathbb{R}^{m} \rightarrow[0, \infty)$ be a convex function and let $w \in L_{\mathrm{loc}}^{1}\left(\mathbb{R}^{n}, \mathbb{R}^{m}\right)$. Then, for each bounded open sets $\Omega^{\prime}$ and $\Omega$ with $\Omega^{\prime} \Subset \Omega$, for each $0<\epsilon<\operatorname{dist}\left(\Omega^{\prime}, \mathbb{R}^{n} \backslash \Omega\right)$,

$$
\int_{\Omega^{\prime}} f\left(\rho_{\epsilon} * w\right) d x \leq \int_{\Omega} f(w) d x
$$

Proof. (i) See, for instance, [DM, Proof of Theorem 23.1]

(ii) See, for instance, DM, (23.5)].

2.3. Approximation by piecewise affine functions. It is well known (see, for instance, [ET, Chap. X, Proposition 2.9]) that the class of piecewise affine functions is dense in the classical Sobolev space $W^{1, p}(\Omega)$, provided that $\Omega$ is a bounded open set with Lipschitz boundary. This result is crucial in the proof of the classical integral representation theorem with respect to the Euclidean gradient (see, for instance, [DM, Theorem 20.1]). The aim of this section is to prove that no results of this kind are available for a general family $X=\left(X_{1}, \ldots, X_{m}\right)$ in $\mathbb{R}^{n}$, by extending, in a natural way, the notion to be affine with respect to the $X$-gradient. We say that $u \in \mathbf{C}^{\infty}\left(\mathbb{R}^{n}\right)$ is $X$-affine if there exists $c \in \mathbb{R}^{n}$ such that $X u(x)=c$ for all $x \in \mathbb{R}^{n}$. Let $\Omega \subset \mathbb{R}^{n}$ be open. We say that $u: \Omega \rightarrow \mathbb{R}$ is $X$-affine if it is the restriction to $\Omega$ of a $X$-affine function over $\mathbb{R}^{n}$. Moreover, we say that $u: \mathbb{R}^{n} \rightarrow \mathbb{R}$ is $X$-piecewise affine if it is continuous and there is a partition of $\mathbb{R}^{n}$ into a negligible set and a finite number of open sets on which $u$ is $X$-affine. We prove that for Grushin and Heisenberg vector fields the approximation of functions in $W_{X}^{1, p}(\Omega)$ using $X$-piecewise affine functions does not hold. 
It is easy to see that, if $X=\left(X_{1}, X_{2}\right)$ is the Heisenberg vector field on $\mathbb{R}^{3}$ (see Example 2.2 (iii)), then a function $u \in \mathbf{C}^{\infty}\left(\mathbb{R}^{3}\right)$ is $X$-affine if and only if

$$
u(x)=c_{1} x_{1}+c_{2} x_{2}+c_{3} \text { for each } x=\left(x_{1}, x_{2}, x_{3}\right) \in \mathbb{R}^{3},
$$

for suitable constants $c_{i} \in \mathbb{R} i=1,2,3$. Indeed, it is trivial that a function $u$ in (19) is $X$ - affine. Conversely, if $X_{1} u=c_{1}$ and $X_{2} u=c_{2}$ on $\mathbb{R}^{3}$, for some $u \in \mathbf{C}^{\infty}\left(\mathbb{R}^{3}\right)$, then the commutator $\left[X_{1}, X_{2}\right] u:=\left(X_{1} X_{2}-X_{2} X_{1}\right) u=\partial_{3} u=$ 0 on $\mathbb{R}^{3}$, which gives $u(x)=c_{1} x_{1}+c_{2} x_{2}+c_{3}$ for each $x=\left(x_{1}, x_{2}, x_{3}\right) \in \mathbb{R}^{3}$, for some $c_{3} \in \mathbb{R}$.

Let $u(x)=x_{3}$, then $u \in W_{X}^{1, p}(\Omega)$ whenever $|\Omega|<\infty$. Since any $X$-piecewise affine function does not depend on $x_{3}$, there cannot be any sequence of $X$-piecewise affine functions $\left(u_{h}\right)_{h}$ such that $u_{h}\left(x_{1}, x_{2}, x_{3}\right) \rightarrow u\left(x_{1}, x_{2}, x_{3}\right)$ for a.e. $\left(x_{1}, x_{2}, x_{3}\right) \in \Omega$.

Let $X=\left(X_{1}, X_{2}\right)$ be the Grushin vector fields on $\mathbb{R}^{2}$ (see Example 2.2 (ii)). Let $u \in \mathbf{C}^{\infty}\left(\mathbb{R}^{2}\right)$ be such that $X_{1} u=c_{1}$ and $X_{2} u=c_{2}$ on $\mathbb{R}^{2}$. Then it is easy to prove, arguing as before, that $u(x)=c_{1} x_{1}+c_{3}$ for each $x=\left(x_{1}, x_{2}\right) \in \mathbb{R}^{2}$, for some $c_{3} \in \mathbb{R}$. The conclusion follows as in the previous case taking $u\left(x_{1}, x_{2}\right)=x_{2}$, which belongs to $W_{X}^{1, p}(\Omega)$ for any $p \geq 1$ and any bounded open set $\Omega \subset \mathbb{R}^{2}$.

\section{Relaxation AND CHARACTERIZATION OF INTEGRAL FUnCtionals DEPENDING ON VECTOR FIELDS}

In the study of the $\Gamma$-convergence it will be helpful to consider $F$ and $F_{1}$ as local functionals. Namely, according to [DM, Chap. 15], we will consider the functionals $F, F_{1}: L^{p}(\Omega) \times \mathcal{A} \rightarrow[0, \infty]$

$$
F(u, A):= \begin{cases}\int_{A} f(x, X u(x)) d x & \text { if } A \in \mathcal{A} \text { and } u \in \mathbf{C}^{1}(A) \cap L^{p}(A) \\ \infty & \text { otherwise }\end{cases}
$$

$$
F_{1}(u, A):= \begin{cases}\int_{A} f(x, X u(x)) d x & \text { if } A \in \mathcal{A} \text { and } u \in W_{\mathrm{loc}}^{1,1}(A) \cap L^{p}(A) \\ \infty & \text { otherwise }\end{cases}
$$

For future use, we denote by $\mathcal{A}_{0}$ the class of all open sets compactly contained in $\Omega$.

\subsection{Characterization of the relaxed functional and its finiteness} domain. We are going to characterize the relaxed functionals of $F$ in (11) and $F_{1}$ in (2) with respect to the topology of $L^{p}(\Omega)$. Let us recall that the relaxed functional of a given functional $G: L^{p}(\Omega) \rightarrow[0, \infty]$ is defined as follows (see, for instance, [B]):

$$
\bar{G}(u):=\inf \left\{\liminf _{h \rightarrow \infty} G\left(u_{h}\right):\left(u_{h}\right)_{h} \subset L^{p}(\Omega), u_{h} \rightarrow u \text { in } L^{p}(\Omega)\right\} .
$$


Then it is well known (see, for instance, [B] ) that $\bar{G}$ is the greatest $L^{p}(\Omega)$ lower semicontinuous functional smaller or equal to $G$.

The relaxed functionals $\bar{F}$ and $\bar{F}_{1}$ can be characterized as follows:

3.1. Theorem. Let $p>1$ and let $\Omega$ be an open subset of $\mathbb{R}^{n}$; let $f: \Omega \times \mathbb{R}^{m} \rightarrow$ $[0, \infty)$ be an integrand function in $I_{m, p}\left(\Omega, c_{1}, c_{0}\right)$ with $c_{1} \geq c_{0}>0$. Then

(i) $\operatorname{dom} \bar{F}:=\left\{u \in L^{p}(\Omega): \bar{F}(u)<\infty\right\}=W_{X}^{1, p}(\Omega)$;

(ii) $\bar{F}(u)=\int_{\Omega} f(x, X u(x)) d x$ for every $u \in W_{X}^{1, p}(\Omega)$;

(iii) $\bar{F}(u)=\bar{F}_{1}(u)$ for each $u \in L^{p}(\Omega)$.

Proof. Claims (i) and (ii) are proved in EFSC1, Theorem 3.3.1]. Let us prove (iii). Let $u \in L^{p}(\Omega)$ and $\left(u_{h}\right)_{h} \subset \mathbf{C}^{1}(\Omega) \cap L^{p}(\Omega)$ with $u_{h} \rightarrow u$ in $L^{p}(\Omega)$. Since, in particular, $\left(u_{h}\right)_{h} \subset W_{\text {loc }}^{1,1}(\Omega) \cap L^{p}(\Omega)$ we get

$$
\bar{F}_{1}(u) \leq \liminf _{h \rightarrow \infty} \bar{F}_{1}\left(u_{h}\right)=\liminf _{h \rightarrow \infty} \int_{\Omega} f\left(x, X u_{h}\right) d x=\liminf _{h \rightarrow \infty} F\left(u_{h}\right)
$$

which implies

$$
\bar{F}_{1}(u) \leq \bar{F}(u) .
$$

Let $F^{*}: L^{p}(\Omega) \rightarrow[0, \infty]$ denote the functional in (5). By [B, Theorem 2.3.1], $F^{*}$ is $L^{p}(\Omega)$-lower semicontinuous. Let $u \in \operatorname{dom}\left(F_{1}\right):=\left\{v \in W_{l o c}^{1,1}(\Omega) \cap\right.$ $\left.L^{p}(\Omega) \mid F_{1}(u)<\infty\right\}$, then, by $\left(I_{3}\right)$, we have

$$
c_{0} \int_{\Omega}|X u|^{p} d x \leq F_{1}(u)<\infty,
$$

thus $u \in W_{X}^{1, p}(\Omega)$ and $\operatorname{dom} F_{1} \subset W_{X}^{1, p}(\Omega)$. This implies $F^{*} \leq F_{1}$ on $L^{p}(\Omega)$ and consequently $F^{*} \leq \bar{F}_{1}$ on $L^{p}(\Omega)$. Using (24) and (ii) we conclude

$$
F^{*} \leq \bar{F}_{1} \leq \bar{F}=F^{*} \quad \text { on } \quad L^{p}(\Omega)
$$

which completes the proof.

When $p=1$ the domain of relaxed functional $\bar{F}$ gives rise to the space of functions of bounded variation function associated to $X, B V_{X}(\Omega)$ (see [FSSC1, Theorem 3.2.3]).

3.2. A characterization of functionals depending on vector fields. We are going to study when a local functional $F: \mathbf{C}^{1}(\Omega) \times \mathcal{A} \rightarrow[0, \infty]$ can be equivalently represented both with respect to a family of vector fields $X$ and the Euclidean gradient $D$.

We already stressed that the functional $F$ in (11) can be always represented with respect to the Euclidean gradient on $\mathbf{C}^{1}(\Omega)$ by means of the Euclidean integrand (44). 
Then, it is clear that, for each $A \in \mathcal{A}$ and $u \in \mathbf{C}^{1}(A)$,

$$
\begin{aligned}
F(u, A) & =\int_{A} f(x, X u) d x=\int_{A} f(x, C(x) D u) d x \\
& =\int_{A} f_{e}(x, D u) d x .
\end{aligned}
$$

Viceversa, we are going to study when, given a $X$-gradient and a functional $F: \mathbf{C}^{1}(\Omega) \times \mathcal{A} \rightarrow[0, \infty]$

$$
F(u, A)=\int_{A} f_{e}(x, D u) d x \quad u \in \mathbf{C}^{1}(A),
$$

there exist a function $f: \Omega \times \mathbb{R}^{m} \rightarrow[0, \infty]$ such that

$$
F(u, A)=\int_{A} f(x, X u) d x .
$$

Let us begin with some preliminaries of linear algebra.

In the following, we identify the space of real matrices of order $m \times n$ with $\mathbb{R}^{m n}$ or $\mathcal{L}\left(\mathbb{R}^{m}, \mathbb{R}^{n}\right)$, where $\mathcal{L}\left(\mathbb{R}^{m}, \mathbb{R}^{n}\right)$ denotes the class of linear maps from $\mathbb{R}^{m}$ to $\mathbb{R}^{n}$ endowed with its operator norm. Given a matrix $A=\left[a_{i j}\right]$ of order $m \times n$ its operator norm is defined as

$$
\|A\|:=\sup _{|z|=1}|A z|
$$

and its Hilbert-Schmidt norm as

$$
\|A\|_{\mathbb{R}^{m n}}:=\sqrt{\sum_{i, j} a_{i j}^{2}}
$$

(see [La, Chap. 7]). Since the norms are equivalent, we can also identify the spaces

$$
\mathbf{C}^{0}\left(\Omega_{X}, \mathbb{R}^{m n}\right) \equiv \mathbf{C}^{0}\left(\Omega_{X}, \mathcal{L}\left(\mathbb{R}^{m}, \mathbb{R}^{n}\right)\right),
$$

where we recall that $\Omega_{X}=\Omega \backslash \mathcal{N}_{X}$. For each $x \in \Omega$, let $L_{x}: \mathbb{R}^{n} \rightarrow \mathbb{R}^{m}$ be the linear map

$$
L_{x}(v):=C(x) v \text { if } v \in \mathbb{R}^{n}
$$

where $C(x)$ denotes the matrix in (3). Let $N_{x}$ and $V_{x}$ respectively denote the subspaces of $\mathbb{R}^{n}$ defined as

$$
N_{x}:=\operatorname{ker}\left(L_{x}\right), \quad V_{x}:=\left\{C(x)^{T} z: z \in \mathbb{R}^{m}\right\} .
$$

It is well-known that $N_{x}$ and $V_{x}$ are orthogonal complements in $\mathbb{R}^{n}$, that is

$$
\mathbb{R}^{n}=N_{x} \oplus V_{x} .
$$

Moreover, for each $x \in \Omega$ and $\xi \in \mathbb{R}^{n}$, let us define $\xi_{N_{x}} \in N_{x}$ and $\xi_{V_{x}} \in V_{x}$ as the unique vectors of $\mathbb{R}^{n}$ such that

$$
\xi=\xi_{N_{x}}+\xi_{V_{x}}
$$


and let $\Pi_{x}: \mathbb{R}^{n} \rightarrow V_{x} \subset \mathbb{R}^{n}$ be the projection

$$
\Pi_{x}(\xi):=\xi_{V_{x}} .
$$

3.2. Proposition. Assume that the family $X$ of vector fields satisfies (LIC) on $\Omega$. Let $C(x)$ be the matrix in (3) and $L_{x}$ be the map in (28). Then $L_{x}: V_{x} \rightarrow \mathbb{R}^{m}$ is invertible and the map $L^{-1}: \Omega_{X} \rightarrow \mathcal{L}\left(\mathbb{R}^{m}, \mathbb{R}^{n}\right)$ defined as

$$
L^{-1}(x):=L_{x}^{-1} \text { if } x \in \Omega_{X}
$$

belongs to $\mathbf{C}^{0}\left(\Omega_{X}, \mathcal{L}\left(\mathbb{R}^{m}, \mathbb{R}^{n}\right)\right)$.

Before giving the proof of Proposition 3.2, let us prove a preliminary technical lemma.

3.3. Lemma. Under the same assumptions of Proposition 3.2,

(i) $\operatorname{dim} V_{x}=m$ for each $x \in \Omega_{X}$ and $L_{x}\left(V_{x}\right)=\operatorname{range}\left(L_{x}\right)=\mathbb{R}^{m}$ where range $\left(L_{x}\right)$ denotes the range of $L_{x}$, that is, range $\left(L_{x}\right):=\left\{L_{x}(v)\right.$ : $\left.v \in \mathbb{R}^{n}\right\}$. In particular $L_{x}: V_{x} \rightarrow \mathbb{R}^{m}$ is an isomorphism.

(ii) Let

$$
B(x):=C(x) C^{T}(x) \quad x \in \Omega .
$$

Then, for each $x \in \Omega_{X}, B(x)$ is a symmetric invertible matrix of order $m$. Moreover the map $B^{-1}: \Omega_{X} \rightarrow \mathcal{L}\left(\mathbb{R}^{m}, \mathbb{R}^{m}\right)$, defined as

$$
B^{-1}(x)(z):=B(x)^{-1} z \quad \text { if } z \in \mathbb{R}^{m},
$$

is continuous.

(iii) For each $x \in \Omega_{X}$, the projection $\Pi_{x}$ in (32) can be represented as

$$
\Pi_{x}(\xi)=\xi_{V_{x}}=C(x)^{T} B(x)^{-1} C(x) \xi, \quad \forall \xi \in \mathbb{R}^{n} .
$$

If $m=n$, then, $\Pi_{x}=\operatorname{Id}_{n}: \mathbb{R}^{n} \rightarrow \mathbb{R}^{n}$, the identity map in $\mathbb{R}^{n}$.

3.4. Remark. Using the definition of $V_{x}$ it is easy to see that

$$
V_{x}=\operatorname{span}_{\mathbb{R}}\left\{X_{1}(x), \ldots, X_{m}(x)\right\},
$$

i.e., the so-called horizontal bundle, denoted also by $H_{x}$.

Proof. (i) The claim is a well-known result of basic linear algebra.

(ii) It is straightforward that $B(x)$ a symmetric matrix of order $m$ for each $x \in \Omega$. We have only to show that it is invertible for each $x \in \Omega_{X}$ or, equivalently, that

$$
\text { if } B(x) z=0 \text { for some } z \in \mathbb{R}^{m} \text {, then } z=0 .
$$

Let $z^{T}$ denotes the transpose of a column vector $z \in \mathbb{R}^{m}$. If $B(x) z=0$, then

$$
\begin{aligned}
0=z^{T} B(x) z & =z^{T} C(x) C^{T}(x) z \\
& =\left|C^{T}(x) z\right|_{\mathbb{R}^{m}}^{2} \quad \Longleftrightarrow \quad C^{T}(x) z=0 .
\end{aligned}
$$

By (LIC), since

$$
\operatorname{rank} C(x)=\operatorname{rank} C^{T}(x)=m \quad \forall x \in \Omega_{X},
$$


from (37) we get that $z=0$ and (36) follows. Let us now prove that the map (35) is continuous. Let us recall that, given a matrix $A \in \mathbf{C}^{0}\left(\Omega_{X}, \mathbb{R}^{m^{2}}\right)$, by the definition of determinant (see, for instance, [La, Chap.3,Theorem 6]), the determinant map

$$
\operatorname{det} A: \Omega_{X} \rightarrow \mathbb{R}, \quad(\operatorname{det} A)(x):=\operatorname{det}(A(x))
$$

is continuous. Moreover

$$
A(x) \text { is invertible } \Longleftrightarrow \operatorname{det} A(x) \neq 0 .
$$

By Cramer's rule (see, for instance, [La, Chap.3, Theorem 7]), if $B(x)^{-1}=$ $\left[b_{i j}^{*}(x)\right]$, then

$$
b_{i j}^{*}(x)=(-1)^{i+j} \frac{\operatorname{det} B_{i j}(x)}{\operatorname{det} B(x)} \quad x \in \Omega_{X}, i, j=1, \ldots, m,
$$

where $B_{i j}$ is the $(m-1) \times(m-1)$ matrix obtained by striking out the $i$ th row and $j$ th column of $B$, i.e., the $(i j)$ th minor of $B$. This implies that $B^{-1} \in \mathbf{C}^{0}\left(\Omega_{X}, \mathbb{R}^{m^{2}}\right) \equiv \mathbf{C}^{0}\left(\Omega_{X}, \mathcal{L}\left(\mathbb{R}^{m}, \mathbb{R}^{m}\right)\right)$.

(iii) We have

$$
\Pi_{x}(\xi)=\xi_{V_{x}}=C(x)^{T} w
$$

for a suitable (unique) $w=w(x, \xi) \in \mathbb{R}^{m}$ depending on $x$ and $\xi$. On the other hand, by (38),

$$
\begin{aligned}
C(x) \xi & =L_{x}(\xi)=L_{x}\left(\xi_{N_{x}}\right)+L_{x}\left(\xi_{V_{x}}\right) \\
& =C(x) \xi_{V_{x}}=C(x) C(x)^{T} w=B(x) w .
\end{aligned}
$$

Since $B(x)$ is invertible, by (39), we get the desired conclusion.

Proof of Proposition 3.2. The fact that the map $L_{x}: V_{x} \rightarrow \mathbb{R}^{m}$ is invertible follows from Lemma 3.3 (i). Let us now prove that

$$
L_{x}^{-1}(z)=C^{T}(x) B(x)^{-1} z \quad \forall z \in \mathbb{R}^{m},
$$

where $B(x)$ is the matrix in (34). Let us fix $z \in \mathbb{R}^{m}$ and let $v=L_{x}^{-1}(z) \in V_{x}$. By Lemma 3.3 (iii), there exists $w \in \mathbb{R}^{m}$ such that $v=C^{T}(x) w$. Thus

$$
z=L_{x}(v)=C(x) C^{T}(x) w=B(x) w .
$$

By Lemma 3.3 (ii), it holds $w=B(x)^{-1} z$. Therefore we get

$$
L_{x}^{-1}(z)=v=C^{T}(x) B(x)^{-1} z
$$

and (40) follows. Let us define

$$
A(x):=C^{T}(x) B(x)^{-1} \quad \text { if } x \in \Omega_{X} .
$$

Then, from Lemma 3.3 (ii), $A \in \mathbf{C}^{0}\left(\Omega_{X}, \mathbb{R}^{m n}\right) \equiv \mathbf{C}^{0}\left(\Omega_{X}, \mathcal{L}\left(\mathbb{R}^{m}, \mathbb{R}^{n}\right)\right)$. Thus, by (41), we get the desired conclusion. 
3.5. Theorem. Let $\Omega \subset \mathbb{R}^{n}$ be an open set and assume that $X$ satisfies (LIC) on $\Omega$. Let $F: \mathbf{C}^{1}(\Omega) \times \mathcal{A} \rightarrow[0, \infty]$ be the functional in (26) with $f_{e}: \Omega \times \mathbb{R}^{n} \rightarrow[0, \infty]$ a Borel measurable function satisfying

$$
\text { for each } \xi \in \mathbb{R}^{n}, f_{e}(\cdot, \xi) \in L_{\text {loc }}^{1}(\Omega)
$$

and

$$
f_{e}(x, \cdot): \mathbb{R}^{n} \rightarrow[0, \infty) \text { convex for a.e. } x \in \Omega .
$$

Define $f: \Omega \times \mathbb{R}^{m} \rightarrow[0, \infty)$ as

$$
f(x, \eta):= \begin{cases}f_{e}\left(x, L^{-1}(x)(\eta)\right) & \text { if }(x, \eta) \in \Omega_{X} \times \mathbb{R}^{m} \\ 0 & \text { otherwise }\end{cases}
$$

where $L^{-1}: \Omega_{X} \rightarrow \mathcal{L}\left(\mathbb{R}^{m}, \mathbb{R}^{n}\right)$ is the map in (33). Then, $f$ is a Borel measurable function satisfying

$$
f(x, \cdot): \mathbb{R}^{m} \rightarrow[0, \infty) \text { convex for a.e. } x \in \Omega .
$$

Moreover,

$$
\begin{aligned}
F(u, A) & =\int_{A} f_{e}(x, D u) d x \\
& =\int_{A} f(x, X u) d x \quad \forall A \in \mathcal{A}, u \in \mathbf{C}^{1}(A)
\end{aligned}
$$

if and only if

$$
f_{e}(x, \xi)=f_{e}\left(x, \Pi_{x}(\xi)\right) \text { for a.e. } x \in \Omega, \forall \xi \in \mathbb{R}^{n},
$$

where $\left\{V_{x}: x \in \Omega_{X}\right\}$ is the distribution of $m$-planes in $\mathbb{R}^{n}$ defined in Proposition 3.2 and $\Pi_{x}: \mathbb{R}^{n} \rightarrow V_{x}$ denotes the projection of $\mathbb{R}^{n}$ on $V_{x}$ in (32).

In addition, the function $f$ for which (46) holds is unique, that is, if there exists another Borel measurable function $f^{*}: \Omega \times \mathbb{R}^{m} \rightarrow[0, \infty)$ satisfying $f^{*}(x, \cdot): \mathbb{R}^{m} \rightarrow[0, \infty)$ convex a.e. $x \in \Omega$ and (46) holds, then $f(x, \eta)=f^{*}(x, \eta)$ for a.e. $x \in \Omega$ and $\eta \in \mathbb{R}^{m}$.

3.6. Remark. If the $X$-gradient does not satisfy (LIC) condition, the uniqueness of representation (46) may trivially fail. For instance, let $X=\left(X_{1}, X_{2}\right):=$ $\left(\partial_{1}, 0\right)$ be the family of vector fields on $\Omega=\mathbb{R}^{2}$ and let $f(\eta):=\eta_{1}^{2}+g\left(\eta_{2}\right)$ and $f^{*}(\eta):=\eta_{1}^{2}+g^{*}\left(\eta_{2}\right)$ for each $\eta=\left(\eta_{1}, \eta_{2}\right) \in \mathbb{R}^{2}$, where $g, g^{*}: \mathbb{R} \rightarrow$ $[0, \infty)$ are convex functions satisfying $g(0)=g^{*}(0)=0$, but $g \neq g^{*}$. Then it clear that $f$ and $f^{*}$ are integrand functions of the same functional $F$ defiined in (46), even though $f \neq f^{*}$.

3.7. Remark. Notice that, in the case $m=n$ and $X$ satisfies (LIC) on $\Omega$, condition (47) always holds, since, by Lemma 3.3 (iii), $\Pi_{x} \equiv \operatorname{Id}_{n}$.

Proof. 1st step. Let us prove that $f$ is Borel measurable. Let $\Psi: \Omega_{X} \times$ $\mathbb{R}^{m} \rightarrow \Omega_{X} \times \mathbb{R}^{n}$ denote the map

$$
\Psi(x, \eta):=\left(x, L^{-1}(x)(\eta)\right) \quad \text { if }(x, \eta) \in \Omega_{X} \times \mathbb{R}^{m} .
$$


By Proposition 3.2, $\Psi$ is continuous, then it is also Borel measurable. Since $f_{e}: \Omega \times \mathbb{R}^{n} \rightarrow[0, \infty]$ is Borel measurable, the composition $f=f_{e} \circ \Psi$ : $\Omega_{X} \times \mathbb{R}^{m} \rightarrow[0, \infty]$ is still Borel measurable.

To prove (45) it is sufficient to notice that

$$
f(x, \cdot)=f_{e}(x, \cdot) \circ L^{-1}(x) \quad \forall x \in \Omega_{X}
$$

indeed $f_{e}(x, \cdot): \mathbb{R}^{n} \rightarrow[0, \infty)$ is convex for a.e. $x \in \Omega$ and $L^{-1}(x): \mathbb{R}^{m} \rightarrow$ $\mathbb{R}^{n}$ is linear for $x \in \Omega_{X}$.

2nd step. Let us prove the uniqueness of representation in (46). Assume that

$$
\begin{aligned}
\int_{A} f(x, X u) d x & =\int_{A} f^{*}(x, X u) d x \\
& =\int_{A} f_{e}(x, D u) d x \quad \forall u \in \mathbf{C}^{1}(A), A \in \mathcal{A}
\end{aligned}
$$

for given Borel measurable functions $f, f^{*}: \Omega \times \mathbb{R}^{m} \rightarrow[0, \infty)$, with $f(x, \cdot), f^{*}(x, \cdot)$ : $\mathbb{R}^{m} \rightarrow[0, \infty)$ convex a.e. $x \in \Omega$. Let us choose as

$$
u(x)=u_{\xi}(x):=\langle\xi, x\rangle \quad x \in \mathbb{R}^{n},
$$

for fixed $\xi \in \mathbb{Q}^{n}$, in the previous equality. By (48) and (42), it follows that the functions

$$
\Omega \ni y \mapsto f(y, C(y) \xi) \text { and } \Omega \ni y \mapsto f^{*}(y, C(y) \xi) \text { are in } L_{\mathrm{loc}}^{1}(\Omega) \text {. }
$$

Choosing $A=B(x, r)$ in (48), by Lebesgue's differentiation theorem, we get that there exists a negligible set $\mathcal{N}_{\xi} \subset \Omega$ such that $\forall x \in \Omega \backslash \mathcal{N}_{\xi}$

$$
\begin{aligned}
f\left(x, L_{x}(\xi)\right) & =f(x, C(x) \xi)=f^{*}(x, C(x) \xi) \\
& =f^{*}\left(x, L_{x}(\xi)\right) .
\end{aligned}
$$

If $\mathcal{N}:=\cup_{\xi \in \mathbb{Q}^{n}} \mathcal{N}_{\xi}$, then (50) holds for each $x \in \Omega \backslash \mathcal{N}$ and $\xi \in \mathbb{Q}^{n}$. Since, for each $x \in \Omega \backslash \mathcal{N}, f(x, \cdot), f^{*}(x, \cdot): \mathbb{R}^{m} \rightarrow[0, \infty)$ are continuous, it follows that (50) holds for each $x \in \Omega \backslash \mathcal{N}$ and $\xi \in \mathbb{R}^{n}$. Being the map $L_{x}: \mathbb{R}^{n} \rightarrow \mathbb{R}^{m}$ onto, we get the desired conclusion.

3nd step. Let us assume (47). To prove (46) it is sufficient to prove that, for each $A \in \mathcal{A}, u \in \mathbf{C}^{1}(A)$

$$
f(x, X u(x))=f_{e}(x, D u(x)) \text { a.e. } x \in \Omega .
$$

Given $A \in \mathcal{A}$ and $u \in \mathbf{C}^{1}(A)$, let us recall that

$$
X u(x)=C(x) D u(x) \quad \forall x \in A .
$$


Thus, by (47), Lemma 3.3 (iii) and the definition of $V_{x}$, a.e. $x \in \Omega$, if $v_{x}:=D u(x)$

$$
\begin{aligned}
f(x, X u(x)) & =f\left(x, C(x) v_{x}\right)=f\left(x, L_{x}\left(\Pi_{x}\left(v_{x}\right)\right)\right) \\
& =f_{e}\left(x, L_{x}^{-1}\left(L_{x}\left(\Pi_{x}\left(v_{x}\right)\right)\right)=f_{e}\left(x, \Pi_{x}\left(v_{x}\right)\right)\right. \\
& =f_{e}\left(x, v_{x}\right)=f_{e}(x, D u(x))
\end{aligned}
$$

and (51) follows. On the other hand, let us assume that for every $A \in \mathcal{A}$ and $u \in \mathbf{C}^{1}(A)$

$$
\int_{A} f_{e}(x, D u) d x=\int_{A} f(x, X u) d x
$$

where $f$ is the function in (44). By (52), for every $A \in \mathcal{A}$ and $u \in \mathbf{C}^{1}(A)$,

$$
f(x, X u(x))=f_{e}\left(x, \Pi_{x}(D u(x))\right) \quad \forall x \in A,
$$

which implies

$$
\int_{A} f(x, X u(x)) d x=\int_{A} f_{e}\left(x, \Pi_{x}(D u(x))\right) d x .
$$

Thus, for every $A \in \mathcal{A}$ and $u \in \mathbf{C}^{1}(A)$,

$$
\int_{A} f_{e}\left(x, \Pi_{x}(D u(x))\right) d x=\int_{A} f_{e}(x, D u) d x
$$

and the conclusion now follows by proceeding as in the second step of the proof.

3.8. Remark. Observe that (51) actually holds for each $u \in W^{1, p}(A)$. As a consequence, (46) holds for each $A \in \mathcal{A}$ and $u \in W^{1, p}(A)$.

3.3. Integral representation for local functionals with respect to vector fields. Let us recall, for reader's convenience, some notation about set functions on $\mathcal{A}$ and local functionals on $L^{p}(\Omega) \times \mathcal{A}$, according to DM. Let $\Omega \subset \mathbb{R}^{n}$ be an open set.

3.9. Definition. Let $\alpha: \mathcal{A} \rightarrow[0, \infty]$ be a set function. We say that:

(i) $\alpha$ is increasing if $\alpha(A) \leq \alpha(B)$, for each $A, B \in \mathcal{A}$ with $A \subseteq B$;

(ii) $\alpha$ is inner regular if

$$
\alpha(A)=\sup \{\alpha(B): B \in \mathcal{A}, B \Subset A\} \text { for each } A \in \mathcal{A} ;
$$

(iii) $\alpha$ is subadditive if $\alpha(A) \leq \alpha\left(A_{1}\right)+\alpha\left(A_{2}\right)$ for every $A, A_{1}, A_{2} \in \mathcal{A}$ with $A \subset A_{1} \cup A_{2}$;

(iv) $\alpha$ is superadditive if $\alpha(A) \geq \alpha\left(A_{1}\right)+\alpha\left(A_{2}\right)$ for every $A, A_{1}, A_{2} \in \mathcal{A}$ with $A_{1} \cup A_{2} \subseteq A$ and $A_{1} \cap A_{2}=\emptyset$;

(v) $\alpha$ is a measure if there exists a Borel measure $\mu: \mathcal{B}(\Omega) \rightarrow[0, \infty]$ such that $\alpha(A)=\mu(A)$ for every $A \in \mathcal{A}$.

3.10. Remark. Let us recall that, if $\alpha: \mathcal{A} \rightarrow[0, \infty]$ is an increasing set function, then it is a measure if and only if it is subadditive, superadditive and inner regular (see [DM, Theorem 14.23]). 
3.11. Definition. Let

$$
F: L^{p}(\Omega) \times \mathcal{A} \rightarrow[0, \infty]
$$

We say that:

(i) $F$ is increasing if, for every $u \in L^{p}(\Omega), F(u, \cdot): \mathcal{A} \rightarrow[0, \infty]$ is increasing as set function;

(i) $F$ is inner regular (on $\mathcal{A}$ ) if it is increasing and, for each $u \in L^{p}(\Omega)$, $F(u, \cdot): \mathcal{A} \rightarrow[0, \infty]$ is innner regular as set function;

(iii) $F$ is a measure, if for every $u \in L^{p}(\Omega), F(u, \cdot): \mathcal{A} \rightarrow[0, \infty]$ is a measure as set function;

(iv) $F$ is local if

$$
F(u, A)=F(v, A)
$$

for each $A \in \mathcal{A}, u, v \in L^{p}(\Omega)$ such that $u=v$ a.e. on $A$;

(v) $F$ is lower semicontinuous (lsc), if for every $A \in \mathcal{A}, F(\cdot, A): L^{p}(\Omega) \rightarrow$ $[0, \infty]$ is lower semicontinuous.

3.12. Theorem. Let $\Omega \subset \mathbb{R}^{n}$ be a bounded open set and assume that $X$ satisfies (LIC) on $\Omega$. Let $p>1$ and

$$
F: L^{p}(\Omega) \times \mathcal{A} \rightarrow[0, \infty]
$$

be an increasing functional satisfying the following properties:

(a) F is local;

(b) $F$ is a measure;

(c) $F$ is lsc;

(d) $F(u+c, A)=F(u, A)$ for each $u \in L^{p}(\Omega), A \in \mathcal{A}$ and $c \in \mathbb{R}$;

(e) there exist a non negative function $a \in L_{\mathrm{loc}}^{1}(\Omega)$ and a positive constant $b$ such that

$$
0 \leq F(u, A) \leq \int_{A}\left(a(x)+b|X u(x)|^{p}\right) d x
$$

for each $u \in \mathbf{C}^{1}(A), A \in \mathcal{A}$.

Then, there exists a Borel function $f: \Omega \times \mathbb{R}^{m} \rightarrow[0, \infty]$ such that:

(i) for each $u \in L^{p}(\Omega)$, for each $A \in \mathcal{A}$ with $\left.u\right|_{A} \in W_{X ; \text { loc }}^{1, p}(A)$, we have

$$
F(u, A)=\int_{A} f(x, X u(x)) d x
$$

(ii) for a.e. $x \in \Omega, f(x, \cdot): \mathbb{R}^{m} \rightarrow[0, \infty)$ is convex;

(iii) for a.e. $x \in \Omega$,

$$
0 \leq f(x, \eta) \leq a(x)+b|\eta|^{p} \quad \forall \eta \in \mathbb{R}^{m} .
$$

In order to prove Theorem 3.12, we need two auxiliary key lemmas.

The former is well-known (see, for instance, [Ro, Theorem 12.1]). Let us recall that an affine function $\varphi: \mathbb{R}^{n} \rightarrow \mathbb{R}$ is a function

$$
\varphi(\xi)=\langle z, \xi\rangle+k \quad \forall \xi \in \mathbb{R}^{n},
$$

for a suitable $z \in \mathbb{R}^{n}$ and $k \in \mathbb{R}$. 
3.13. Lemma. Let $g: \mathbb{R}^{n} \rightarrow \mathbb{R}$ be a convex function. Then

$$
g(\xi)=\sup \left\{\varphi(\xi): \varphi \text { affine, } \varphi(\xi) \leq g(\xi) \quad \forall \xi \in \mathbb{R}^{n}\right\} .
$$

The latter will turn out to be a key result through the paper and provides when a Euclidean integrand can be represented as an integrand respect to $X$-gradient.

3.14. Lemma. Let $f_{e}: \Omega \times \mathbb{R}^{n} \rightarrow[0, \infty]$ be a Borel measurable function. Suppose that

(i) for a.e. $x \in \Omega, f_{e}(x, \cdot): \mathbb{R}^{n} \rightarrow[0, \infty)$ is convex;

(ii) there exist a non negative function $a \in L_{\mathrm{loc}}^{1}(\Omega)$ and a positive constant $b$ such that for a.e. $x \in \Omega$

$$
f_{e}(x, \xi) \leq a(x)+b|C(x) \xi|^{p} \quad \forall \xi \in \mathbb{R}^{n},
$$

where $C(x)$ denotes the coefficient matrix of $X$-gradient in (3).

Then, $f_{e}$ satisfies (47).

Proof. Let us prove that, for a.e. $x \in \Omega$,

$$
f_{e}\left(x, \xi_{N_{x}}+\zeta\right)=f_{e}(x, \zeta) \quad \forall \xi, \zeta \in \mathbb{R}^{n},
$$

according to notation in section 3.2. Notice that (53) is equivalent to (47), that is, for a.e. $x \in \Omega$

$$
f_{e}(x, \xi)=f_{e}\left(x, \xi_{V_{x}}\right) \quad \forall \xi \in \mathbb{R}^{n} .
$$

By our assumptions, we can assume that, for a.e. $x \in \Omega, g:=f_{e}(x, \cdot)$ : $\mathbb{R}^{n} \rightarrow[0, \infty)$ is a convex function and (ii) holds with $a=a(x) \in[0, \infty)$. Let $\varphi: \mathbb{R}^{n} \rightarrow \mathbb{R}$ be affine with $\varphi(\xi)=\langle z, \xi\rangle+k$ and $\varphi(\xi) \leq g(\xi)$ for each $\xi \in \mathbb{R}^{n}$. Let us prove that

$$
\langle z, v\rangle=0 \quad \forall v \in N_{x} .
$$

Let $v \in N_{x} \backslash\{0\}$ be given, then also $t v \in N_{x}$ for each $t \in \mathbb{R}$. In particular, $C(x) t v=0$ for each $t \in \mathbb{R}$. Then, by (ii)

$$
\varphi(t v)=t\langle z, v\rangle+k \leq g(t v) \leq a \quad \forall t \in \mathbb{R} .
$$

The previous inequality implies (54). From (54), we get that

$$
\begin{aligned}
\varphi\left(\xi_{N_{x}}+\zeta\right) & =\left\langle a, \xi_{N_{x}}+\zeta\right\rangle+b \\
& =\langle a, \zeta\rangle+b=\varphi(\zeta) \quad \forall \xi, \zeta \in \mathbb{R}^{n} .
\end{aligned}
$$

From Lemma 3.13, (53) follows.

Proof Theorem 3.12. Let us first observe that inequality in assumption (e) can be extended to each $u \in W_{X}^{1, p}(A), A \in \mathcal{A}$. Let us recall that, if $A \in \mathcal{A}$, by Proposition 2.7, given $\left(\rho_{\epsilon}\right)_{\varepsilon}$ a family of mollifiers, then, for each $u \in W_{X}^{1, p}(A)$, denoting by $\bar{u}$ its extension to $\mathbb{R}^{n}$ being 0 outside $\Omega$, if

$$
u_{\varepsilon}(x):=\bar{u} * \rho_{\varepsilon}(x) \quad x \in \mathbb{R}^{n},
$$


for each $A^{\prime} \in \mathcal{A}$ with $A^{\prime} \Subset A$, we have

$$
\begin{gathered}
u_{\varepsilon} \rightarrow u \text { in } L^{p}(\Omega) ; \\
\left.u_{\varepsilon}\right|_{A^{\prime}} \in W_{X}^{1, p}\left(A^{\prime}\right) \text { and } u_{\varepsilon} \rightarrow u \text { in } W_{X}^{1, p}\left(A^{\prime}\right) .
\end{gathered}
$$

Let $u \in L^{p}(\Omega)$ be such that $\left.u\right|_{A} \in W^{1, p}(A)$ for some $A \in \mathcal{A}$. For each $A^{\prime} \Subset A$, by assumption (c), (56) and (57), it follows that

$$
\begin{aligned}
F\left(u, A^{\prime}\right) & \leq \liminf _{\varepsilon \rightarrow 0} F\left(u_{\varepsilon}, A^{\prime}\right) \leq \lim _{\varepsilon \rightarrow 0}\left(\int_{A^{\prime}}\left(a(x)+b\left|X u_{\varepsilon}(x)\right|^{p}\right) d x\right) \\
& =\int_{A^{\prime}}\left(a(x)+b|X u(x)|^{p}\right) d x .
\end{aligned}
$$

Since $F(u, \cdot)$ is a measure, it is also inner regular (see Remark 3.10). Thus, taking the supremum on all $A^{\prime} \in \mathcal{A}$ with $A^{\prime} \Subset A$, we get the desired conclusion. We will now divide the proof in three steps.

1st step. Let us first prove that there exists an integral representation of $F$ with respect to a Euclidean integrand, that is, there exists a Borel function $f_{e}: \Omega \times \mathbb{R}^{n} \rightarrow[0, \infty]$ and a positive constant $b_{2}$ such that

$$
F(u, A)=\int_{A} f_{e}(x, D u) d x
$$

for each $u \in L^{p}(\Omega), A \in \mathcal{A}$ with $\left.u\right|_{A} \in W_{\text {loc }}^{1, p}(A)$;

(61) (47) holds, that is, for a.e. $x \in \Omega f_{e}(x, \xi)=f_{e}\left(x, \Pi_{x}(\xi)\right) \quad \forall \xi \in \mathbb{R}^{n}$.

By (15), if $u \in W^{1 \cdot p}(\Omega)$, then, for a.e. $x \in \Omega$, we have that

$$
|X u(x)|^{p} \leq \sup _{x \in \Omega}\|C(x)\|^{p}|D u(x)|^{p}=b_{2}|D u(x)|^{p},
$$

with $b_{2}<\infty$, since the coefficients of $X$-gradient are Lipschitz on $\Omega$. By (62) and assumption (e), it follows that

$$
0 \leq F(u, A) \leq \int_{A}\left(a(x)+b_{2}|D u(x)|^{p}\right) d x,
$$

for each $u \in W^{1 . p}(\Omega)$, for every $A \in \mathcal{A}$. Therefore by (a), (b), (c), (d) and (63), by applying [DM, Theorem 20.1], there exists a Borel function $f_{e}: \Omega \times \mathbb{R}^{n} \rightarrow[0, \infty]$ satisfying (58), (59) and (60). Observe now that, by (58) and assumption (e), if $u=u_{\xi}$, if follows that, for each $x \in \mathbb{R}^{n}$,

$$
\int_{A} f_{e}(x, \xi) d x \leq \int_{A}\left(a(x)+b|C(x) \xi|^{p}\right) d x \quad \forall A \in \mathcal{A} .
$$


From this integral inequality, arguing as in section 3.2, we can infer the pointwise inequality, that is, there exists a negligible set $\mathcal{N} \subset \Omega$, such that, for each $x \in \Omega \backslash \mathcal{N}$,

$$
f_{e}(x, \xi) \leq a(x)+b|C(x) \xi|^{p} \quad \forall \xi \in \mathbb{R}^{n},
$$

From (59), (64) and Lemma 3.14, (61) holds.

2nd step. Let us prove that there exists a Borel function $f: \Omega \times \mathbb{R}^{m} \rightarrow$ $[0, \infty]$ such that

$$
F(u, A)=\int_{A} f(x, X u) d x,
$$

for each $A \in \mathcal{A}, u \in \mathbf{C}^{1}(A)$ satisfying claims (ii) and (iii). By (59), (60) and (61), we can apply Theorem 3.5 and (65) follows at once with $f: \Omega \times \mathbb{R}^{m} \rightarrow$ $[0, \infty]$ defined as in (44), which also satisfies claim (ii).

From assumption (e) and (65) with $u=u_{\xi}$, it follows that

$0 \leq \int_{A} f(y, C(y) \xi) d y \leq \int_{A}\left(a(y)+b|C(y) \xi|^{p}\right) d y \quad$ for each $A \in \mathcal{A}, \xi \in \mathbb{R}^{n}$.

Taking $A=B(x, r)$, applying Lebesgue's differentiation theorem and arguing as before, from the previous inequality, we can get the following pointwise estimate: for a.e. $x \in \Omega$ it holds that

$$
0 \leq f(x, C(x) \xi) \leq a(x)+b|C(x) \xi|^{p} \quad \forall \xi \in \mathbb{R}^{n} .
$$

Observe now that, by (LIC), for a.e. $x \in \Omega$, the map $L_{x}: \mathbb{R}^{n} \rightarrow \mathbb{R}^{m}$, $L_{x}(\xi):=C(x) \xi$, is surjective. Then claim (iii) also follows.

3rd step. Let us prove that the integral representation in (65) can be extended to functions $u \in W_{X, \text { loc }}^{1, p}(A)$. Therefore claim (i) will follow.

Let us begin to observe that, given $A \in \mathcal{A}_{0}$, the functional

$$
W_{X}^{1, p}(A) \ni u \mapsto \int_{A} f(x, X u) d x \text { is (strongly) continuous. }
$$

Indeed, since for a.e. $x \in \Omega, f(x, \cdot): \mathbb{R}^{m} \rightarrow[0, \infty)$ is continuous and claim (iii) holds, we can apply the Carathéodory continuity theorem (see, for instance, [DM, Example 1.22]).

Let $u \in W_{X}^{1, p}(\Omega)$ and let $A, A^{\prime} \in \mathcal{A}$ with $A^{\prime} \Subset A$. Since $F\left(\cdot, A^{\prime}\right): L^{p}(\Omega) \rightarrow$ $[0, \infty]$, by (56), it follows that

$$
F\left(u, A^{\prime}\right) \leq \liminf _{\varepsilon \rightarrow 0^{+}} F\left(u_{\varepsilon}, A^{\prime}\right)=\lim _{\varepsilon \rightarrow 0^{+}} \int_{A^{\prime}} f\left(x, X u_{\varepsilon}\right) d x=\int_{A^{\prime}} f(x, X u) d x .
$$

As $F$ is a measure, taking the limit as $A^{\prime} \uparrow A$, we get

$$
F(u, A) \leq \int_{A} f(x, X u) d x,
$$

for every $u \in W_{X}^{1, p}(\Omega)$, for each $A \in \mathcal{A}$.

Let us fix $w \in W_{X}^{1, p}(\Omega)$ and let us consider the functional $G: L^{p}(\Omega) \times \mathcal{A} \rightarrow$ $[0, \infty]$

$$
G(u, A):=\underset{20}{F}(u+w, A) .
$$


It is easy to show that $G$ still satisfies assumptions (a)-(e). Thus, by the second step, there exists a Borel function $g: \Omega \times \mathbb{R}^{m} \rightarrow$ satisfying claims (ii) and (iii) with $f \equiv g$, for suitable $a \in L_{\mathrm{loc}}^{1}(\Omega)$ and $b>0$ such that

$$
G(u, A)=\int_{A} g(x, X u) d x,
$$

for each $A \in \mathcal{A}, u \in \mathbf{C}^{1}(A)$ and

$$
G(u, A) \leq \int_{A} g(x, X u) d x,
$$

for every $u \in W_{X}^{1, p}(\Omega)$, for each $A \in \mathcal{A}$. Moreover, arguing as in (66), one can prove that, for each $A \in \mathcal{A}_{0}$, the functional

$$
W_{X}^{1, p}(A) \ni u \mapsto \int_{A} g(x, X u) d x \text { is (strongly) continuous. }
$$

Let

$$
w_{\varepsilon}:=\bar{w} * \rho_{\varepsilon}: \mathbb{R}^{n} \rightarrow \mathbb{R}
$$

and fix $A \in \mathcal{A}$. Then, for every $A^{\prime} \in \mathcal{A}$ with $A^{\prime} \Subset A$, as $\varepsilon \rightarrow 0^{+}$,

$$
w_{\varepsilon} \rightarrow w \text { in } L^{p}(\Omega) \text { and } w_{\varepsilon} \rightarrow w \text { in } W_{X}^{1, p}\left(A^{\prime}\right) .
$$

Thus, by (66), (67), (68), (69), (70) we obtain

$$
\begin{aligned}
\int_{A^{\prime}} g(x, 0) d x & =G\left(0, A^{\prime}\right)=F\left(w, A^{\prime}\right) \leq \int_{A^{\prime}} f(x, X w) d x \\
& =\lim _{\varepsilon \rightarrow 0^{+}} \int_{A^{\prime}} f\left(x, X w_{\varepsilon}\right) d x=\lim _{\varepsilon \rightarrow 0^{+}} F\left(w_{\varepsilon}, A^{\prime}\right) \\
& =\lim _{\varepsilon \rightarrow 0^{+}} G\left(w_{\varepsilon}-w, A^{\prime}\right)=\lim _{\varepsilon \rightarrow 0^{+}} \int_{A^{\prime}} g\left(x, X w_{\varepsilon}-X w\right) d x \\
& =\int_{A^{\prime}} g(x, 0) .
\end{aligned}
$$

This implies that

$$
F\left(w, A^{\prime}\right)=\int_{A^{\prime}} f(x, X w) d x \text { for each } A^{\prime} \in \mathcal{A} \text { with } A^{\prime} \Subset A .
$$

Taking the limit as $A^{\prime} \uparrow A$ in the previous identity, we get that

$$
F(w, A)=\int_{A} f(x, X w) d x \text { for each } w \in W_{X}^{1, p}(\Omega) \text { and } A \in \mathcal{A} .
$$

If $u \in L^{p}(\Omega), A \in \mathcal{A}$ and $\left.u\right|_{A} \in W_{X ; \text { loc }}^{1, p}(A)$ then, for every $A^{\prime} \in \mathcal{A}$ with $A^{\prime} \Subset A$, by Remark 2.6, there exists $w \in W_{X}^{1, p}(\Omega)$ such that

$$
\left.u\right|_{A^{\prime}}=\left.w\right|_{A^{\prime}} .
$$


Since $F$ is local, by (71), we obtain that

$$
F\left(u, A^{\prime}\right)=F\left(w, A^{\prime}\right)=\int_{A^{\prime}} f(x, X w) d x=\int_{A^{\prime}} f(x, X u) d x .
$$

Taking the limit as $A^{\prime} \uparrow A$ we get

$$
F(u, A)=\int_{A} f(x, X u) d x,
$$

which concludes the proof.

3.15. Counterexample. If $X$ agrees with the Euclidean gradient (Example 2.2 (i)), there are well-known examples that, dropping one of the assumptions among (a)-(e) in Theorem 3.12, then the conclusion may fail (see, for instance, $[\mathrm{B}]$ ). Let $X$ be the Heisenberg vector fields in $\mathbb{R}^{3}$ (Example 2.2 (iii)), let $\Omega \subset \mathbb{R}^{3}$ be a bounded open set containing the origin and $p=2$. Then we give an instance that, dropping assumption (e), the conclusion of Theorem 3.12 may fail. Let $F: L^{2}(\Omega) \times \mathcal{A} \rightarrow[0, \infty]$ be the local functional defined as

$$
F(u, A):=\left\{\begin{array}{ll}
\int_{A}|D u|^{2} d x & \text { if } u \in W^{1,2}(A) \\
\infty & \text { otherwise }
\end{array} .\right.
$$

Then, it is clear that $F$ satisfies (a)-(d). Let us prove that functional $F$ cannot satisfy claim (i). Indeed, by contradiction, if there is some integrand $f: \Omega \times \mathbb{R}^{2} \rightarrow[0, \infty]$ for which (i) holds, then, by Theorem 3.5, the compatibility condition (47) must be satisfied, that is,

$$
|\xi|^{2}=f_{e}(x, \xi)=f(x, C(x) \xi)=f_{e}\left(x, \Pi_{x}(\xi)\right)=\left|\Pi_{x}(\xi)\right|^{2}
$$

for a.e. $x \in \Omega, \forall \xi \in \mathbb{R}^{3}$. Since, by Lemma 3.3 (iii), function $\Omega \ni x \mapsto \Pi_{x}(\xi)$ is continuous, the previous identity must hold for each $x \in \Omega$ and $\xi \in \mathbb{R}^{3}$. Let $x=0$, then a simple calculation yields that $\Pi_{0}(\xi)=\left(\xi_{1}, \xi_{2}, 0\right)$ for each $\xi=\left(\xi_{1}, \xi_{2}, \xi_{3}\right) \in \mathbb{R}^{3}$. Thus, if we choose $\xi=(0,0,1)$, the previous identity is not satisfied and then we have a contradiction. This example also shows that the correspondence which maps integrand $f(x, \eta)$ to Euclidean integrand $f_{e}(x, \xi):=f(x, C(x) \xi)$ cannot be reversed.

\section{4. $\Gamma$-CONVERGENCE FOR INTEGRAL FUNCTIONALS DEPENDING ON VECTOR FIELDS}

In this section we are going to show some results concerning $\Gamma$-convergence of integral functionals depending on vector fields, in the strong and weak topology of $W_{X}^{1, p}(\Omega)$ and in the strong one of $L^{p}(\Omega)$. In particular, we will prove a $\Gamma$-compactness result for a class of integral functionals depending on vector fields with respect to $L^{p}(\Omega)$-topology (see Theorem 4.11).

Let us first recall some notions and results concerning $\Gamma$-convergence theory, which are contained in the fundamental monograph [DM] and to which we will refer through this section. We also recommend monograph [Bra] as exastuive account on this topic, containing also interesting applications of $\Gamma$-convergence. 
Let $(X, \tau)$ be a topological space and let $\left(F_{h}\right)_{h}$ be a sequence of functionals from the space $(X, \tau)$ to $\overline{\mathbb{R}}$. Let $\mathcal{U}(x)$ be the family of open neighborhoods of $x \in X$. Then we pose for every $x \in X$

$$
\begin{aligned}
& \left(\Gamma(\tau)-\liminf _{h \rightarrow \infty} F_{h}\right)(x)=\sup _{U \in \mathcal{U}(x)} \liminf _{h \rightarrow \infty} \inf _{U} F_{h} . \\
& \left(\Gamma(\tau)-\limsup _{h \rightarrow \infty} F_{h}\right)(x)=\sup _{U \in \mathcal{U}(x)} \limsup _{h \rightarrow \infty} \inf _{U} F_{h} .
\end{aligned}
$$

They are called, respectively, the $\Gamma$-lower limit and $\Gamma$-upper limit of the sequence $\left(F_{h}\right)_{h}$ in the topology $\tau$.

Then, we give the following definition.

4.1. Definition. Let $\left(F_{h}\right)_{h}$ and $F$ be functionals from space $(X, \tau)$ to $\overline{\mathbb{R}}$. We say that $\left(F_{h}\right)_{h} \Gamma(\tau)$-converges to $F$, or also that $\left(F_{h}\right)_{h} \Gamma$-converges to $F$ in the topology $\tau$, at $x \in X$, if

$$
\left(\Gamma(\tau)-\liminf _{h \rightarrow \infty} F_{h}\right)(x)=\left(\Gamma(\tau)-\limsup _{h \rightarrow \infty} F_{h}\right)(x)=F(x)
$$

and we write

$$
F(x)=\left(\Gamma(\tau)-\lim _{h \rightarrow \infty} F_{h}\right)(x) .
$$

Let us recall below some relevant properties concerning $\Gamma$-convergence that we will need later.

4.2. Theorem. Let $F_{h}$ and $F$ be functionals from space $(X, \tau)$ to $\overline{\mathbb{R}}$.

(i) ([DM, Proposition 6.1]) If $\left(F_{h}\right)_{h} \Gamma(\tau)$-converges to $F$, then each of its subsequence $\left(F_{h_{k}}\right)_{k}$ still $\Gamma(\tau)$-converges to $F$.

(ii) ([DM, Proposition 6.3]) Let $\tau_{i}, i=1,2$, be two topologies on $X$ and suppose that $\tau_{1}$ is weaker than $\tau_{2}$. If $\left(F_{h}\right)_{h} \Gamma\left(\tau_{1}\right)$-converges to $F_{1}$ and $\Gamma\left(\tau_{2}\right)$-converges to $F_{2}$, then $F_{1} \leq F_{2}$.

(iii) ([DM, Theorem 7.8]) (Fundamental Theorem of $\Gamma$-convergence) Assume that the sequence $\left(F_{h}\right)_{h}$ is equicoercive (on $\left.X\right)$, that is, for each $t \in \mathbb{R}$ there exists a closed countably compact $K_{t} \subset X$ such that

$$
\left\{x \in X: F_{h}(x) \leq t\right\} \subset K_{t} \quad \text { for each } h .
$$

Let us also assume that $\left(F_{h}\right)_{h} \Gamma(\tau)$-converges to $F$. Then $F$ is coercive and

$$
\min _{x \in X} F(x)=\lim _{h \rightarrow \infty} \inf _{x \in X} F_{h}(x) .
$$

(iv) ([DM, Proposition 8.1]) Assume that $(X, \tau)$ satisfies the first countability axiom. Then $\left(F_{h}\right)_{h} \Gamma(\tau)$-converges to $F$ if and only if the following two conditions hold:

(1) $(\Gamma-\lim$ inf inequality) for any $x \in X$ and for any sequence $\left(x_{h}\right)_{h}$ converging to $x$ in $X$ one has

$$
F(x) \leq \liminf _{h \rightarrow \infty} F_{h}\left(x_{h}\right) ;
$$


(2) ( $\Gamma-\lim$ equality) for any $x \in X$, there exists a sequence $\left(x_{h}\right)_{h}$ converging to $x$ in $X$ such that

$$
F(x)=\lim _{h \rightarrow \infty} F_{h}\left(x_{h}\right) .
$$

(v) ([DM, Theorem 8.5]) Assume that $(X, \tau)$ satisfies the second countability axiom, that is, there is a countable base for the topology $\tau$. Then every sequence $\left(F_{h}\right)_{h}$ of functionals from $X$ to $\overline{\mathbb{R}}$ has a $\Gamma(\tau)$ convergent subsequence.

4.3. Remark. It is well-known that inequality in Theorem 4.2 (ii) can be strict, even in the case of a (infinite dimensional) Banach space $X, \tau_{1} \equiv$ weak topology of $X$ and $\tau_{2} \equiv$ strong topology of $X$ (see, for instance, [DM, Example 6.6]). An instance of such a phenomenon can occur in the case of non-coercive quadratic integral functionals [ACM].

4.4. Definition $\left(\bar{\Gamma}\right.$-convergence for local functional on $\left.L^{p}(\Omega) \times \mathcal{A}\right)$. Let $F_{h}$ : $L^{p}(\Omega) \times \mathcal{A} \rightarrow[0, \infty](h=1,2, \ldots)$ be a sequence of increasing functionals. We say that the sequence $\left(F_{h}\right)_{h} \bar{\Gamma}$-converges to a functional $F: L^{p}(\Omega) \times \mathcal{A} \rightarrow$ $[0, \infty]$, and we will write $F=\bar{\Gamma}-\lim _{h \rightarrow \infty} F_{h}$, if $F$ is increasing, inner regular and lsc and the following conditions are satisfied:

$[\bar{\Gamma}-\lim$ inf inequality $]$ for each $u \in L^{p}(\Omega)$, for every $A \in \mathcal{A}$ and $\left(u_{h}\right)_{h} \subset$ $L^{p}(\Omega)$ converging to $u$ in $L^{p}(\Omega)$, it holds

$$
F(u, A) \leq \liminf _{h \rightarrow \infty} F_{h}\left(u_{h}, A\right) ;
$$

$\left[\bar{\Gamma}-\limsup\right.$ inequality] for each $u \in L^{p}(\Omega)$, for each $A, B \in \mathcal{A}$ with $A \Subset B$, there exists $\left(u_{h}\right)_{h} \subset L^{p}(\Omega)$ converging to $u$ in $L^{p}(\Omega)$ with

$$
F(u, B) \geq \limsup _{h \rightarrow \infty} F_{h}\left(u_{h}, A\right) .
$$

4.5. Remark. Let us consider a sequence of increasing functionals $F_{h}$ : $L^{p}(\Omega) \times \mathcal{A} \rightarrow[0, \infty](h=1,2, \ldots)$. Assume that there exists a measure functional $F: L^{p}(\Omega) \times \mathcal{A} \rightarrow[0, \infty]$ such that $\left(F_{h}(\cdot, A)\right)_{h} \Gamma$-converges to $F(\cdot, A)$ for each $A \in \mathcal{A}$. Then $\left(F_{h}\right)_{h} \bar{\Gamma}$-converges to $F$. Indeed, being $F$ a $\Gamma$-limit, it is lsc (see [DM, Propostion 6.8]) and it is increasing and inner regular, because it is a measure. Moreover the $\bar{\Gamma}-\lim$ inf and $\bar{\Gamma}-\lim \sup$ inequalities immediately follows by the characterization of $\Gamma$-limit in Theorem 4.2 (iv).

4.6. Definition. Let $F: L^{p}(\Omega) \times \mathcal{A} \rightarrow[0, \infty]$ be a non-negative functional. We say that $F$ satisfies the fundamental estimate if, for every $\varepsilon>0$ and for every $A^{\prime}, A^{\prime \prime}, B \in \mathcal{A}$, with $A^{\prime} \Subset A^{\prime \prime}$, there exists a constant $M>0$ with the following property: for every $u, v \in L^{p}(\Omega)$, there exists a function $\varphi \in \mathbf{C}_{c}^{\infty}\left(A^{\prime \prime}\right)$, with $0 \leq \varphi \leq 1$ on $A^{\prime \prime}, \varphi=1$ in a neighborhood of $A^{\prime}$, such that

$$
\begin{gathered}
F\left(\varphi u+(1-\varphi) v, A^{\prime} \cup B\right) \leq(1+\varepsilon)\left(F\left(u, A^{\prime \prime}\right)+F(v, B)\right)+ \\
+\varepsilon\left(\|u\|_{L^{p}(S)}^{p}+\|v\|_{L^{p}(S)}^{p}+1\right)+M\|u-v\|_{L^{p}(S)},
\end{gathered}
$$


where $S=\left(A^{\prime \prime} \backslash A^{\prime}\right) \cap B$. Moreover, if $\mathcal{F}$ is a class of non-negative functional on $L^{p}(\Omega) \times \mathcal{A}$, we say that the fundamental estimate holds uniformly in $\mathcal{F}$ if each element $F$ of $\mathcal{F}$ satisfies the fundamental estimate with $M$ depending only on $\varepsilon, A^{\prime}, A^{\prime \prime}, B$ while $\varphi$ may depend also on $F, u, v$.

4.7. Remark. Let us recall that, if $F=\bar{\Gamma}-\lim _{h \rightarrow \infty} F_{h}$ and $F_{h}: L^{p}(\Omega) \times \mathcal{A} \rightarrow$ $[0, \infty]$ are measures, then $F$ need not be a measure (see [DM, Examples 16.13 and 16.14]). If the sequence $\left(F_{h}\right)_{h}$ satisfies the fundamental estimates uniformly with respect to $h$, then $F$ is a measure (see [DM, Theorem 18.5]).

Let us now state a result which assures the coincidence between the $\bar{\Gamma}-\lim F_{h}$ and $\Gamma-\lim F_{h}$ for a sequence of local functional $F_{h}: L^{p}(\Omega) \times \mathcal{A} \rightarrow$ $[0, \infty]$, provided that the fundamental estimate holds uniformly for the sequence $\left(F_{h}\right)_{h}$ [DM, Theorem 18.7].

4.8. Theorem. Let $\left(F_{h}\right)_{h}$ be a sequence of non-negative increasing functionals on $L^{p}(\Omega) \times \mathcal{A}$ which $\bar{\Gamma}$-converges to a functional $F$. Assume that there exist two constants $c_{1} \geq 1$ and $c_{2} \geq 0$, a non-negative increasing functional $G: L^{p}(\Omega) \times \mathcal{A} \rightarrow[0, \infty]$, and a non-negative Radon measure $\mu: \mathcal{B}(\Omega) \rightarrow[0, \infty]$ such that

$$
G(u, A) \leq F_{h}(u, A) \leq c_{1} G(u, A)+c_{2}\|u\|_{L^{p}(A)}^{p}+\mu(A)
$$

for every $u \in L^{p}(\Omega), A \in \mathcal{A}$ and $h \in \mathbb{N}$. Assume, in addition, that $G$ is a lower semicontinuous measure and that the fundamental estimate holds uniformly for the sequence $\left(F_{h}\right)_{h}$. Then, $\left(F_{h}(\cdot, A)\right)_{h} \Gamma$-converges in $L^{p}(\Omega)$ to $F(\cdot, A)$ for every $A \in \mathcal{A}$ such that $\mu(A)<\infty$.

4.1. Convergence of integrands and $\Gamma$-convergence for integral functionals depending on vector fields. In this section we will deal with integral functionals $F: W_{X}^{1, p}(\Omega) \rightarrow \mathbb{R}$, with $\Omega$ bounded open subset of $\mathbb{R}^{n}$ and $p>1$, of the form

$$
F(u):=\int_{\Omega} f(x, X u) d x
$$

where the integrand $f: \Omega \times \mathbb{R}^{m} \rightarrow \mathbb{R}$ belongs to class $I_{m, p}\left(\Omega, c_{0}, c_{1}\right)$ (i.e., $f$ satisfies $\left(I_{1}\right),\left(I_{2}\right)$ and $\left(I_{3}\right)$ in the Introduction).

It is easy to show, taking [DM, Proposition 5.12] into account, that the following $\Gamma$-convergence results still hold.

4.9. Proposition. Let $\left(f_{h}\right)_{h}$ and $f$ be functions in $I_{m, p}\left(\Omega, 0, c_{1}\right)$. Let $F_{h}, F$ : $W_{X}^{1, p}(\Omega) \rightarrow \mathbb{R}$ be the corresponding integral functionals in (72). Assume that

$$
F_{h} \rightarrow F\left(\text { pointwise) in } W_{X}^{1 . p}(\Omega) .\right.
$$

Then $\left(F_{h}\right)_{h} \Gamma$-converges to $F$ in $W_{X}^{1 . p}(\Omega)$, i.e.,

$$
F(u)=\left(\Gamma\left(W_{X}^{1, p}(\Omega)\right)-\lim _{\substack{h \rightarrow \infty \\ 25}} F_{h}\right)(u) \quad \forall u \in W_{X}^{1, p}(\Omega) .
$$


The following theorem, in particular, shows that the pointwise convergence of the integrands also implies the $\Gamma$-convergence of the corresponding integral functionals in the weak topology of $W_{X}^{1, p}(\Omega)$.

4.10. Theorem. Let $\left(f_{h}\right)_{h}$ and $f$ be functions in $I_{m, p}\left(\Omega, 0, c_{1}\right)$. Let $F_{h}, F$ : $W_{X}^{1, p}(\Omega) \rightarrow \mathbb{R}$ be the corresponding integral functionals in (72). Assume that

$$
f_{h}(\cdot, \eta) \rightarrow f(\cdot, \eta) \text { a.e. in } \Omega \text {, for each } \eta \in \mathbb{R}^{m} \text {. }
$$

Then

$$
F(u)=\left(\Gamma\left(W_{X}^{1, p}(\Omega) \text {-weak }\right)-\lim _{h \rightarrow \infty} F_{h}\right)(u) \quad \forall u \in W_{X}^{1, p}(\Omega),
$$

i.e., $\left(F_{h}\right)_{h} \Gamma$-converges to $F$ in the weak topology of $W_{X}^{1, p}(\Omega)$.

The scheme of the proof trivially follows the one of [DM, Theorem 5.14] and we omit it.

4.2. $\Gamma$-compactness results for integral functional depending on vector fields. The main result of this section is the following.

4.11. Theorem. Let $\Omega \subset \mathbb{R}^{n}$ be a bounded open set and let $X=\left(X_{1}, \ldots, X_{m}\right)$ satisfy $(L I C)$ on $\Omega$. Let $\left(f_{h}\right)_{h} \subset I_{m, p}\left(\Omega, c_{0}, c_{1}\right)$ and, for each $h$, let $F_{h}^{*}$ : $L^{p}(\Omega) \times \mathcal{A} \rightarrow[0, \infty]$ be the local functional defined as

$$
F_{h}^{*}(u, A):= \begin{cases}\int_{A} f_{h}(x, X u(x)) d x & \text { if } A \in \mathcal{A}, u \in W_{X}^{1, p}(A) \\ \infty & \text { otherwise }\end{cases}
$$

Then, up to a subsequence, there exist a local functional $F: L^{p}(\Omega) \times \mathcal{A} \rightarrow$ $[0, \infty]$ and $f \in I_{m, p}\left(\Omega, c_{0}, c_{1}\right)$ such that

(i) (9) holds;

(ii) F admits the following representation

$$
F(u, A):=\left\{\begin{array}{ll}
\int_{A} f(x, X u(x)) d x & \text { if } A \in \mathcal{A}, u \in W_{X}^{1, p}(A) \\
\infty & \text { otherwise }
\end{array} .\right.
$$

Let us begin to recall a fundamental result about the representantion of the $\bar{\Gamma}$-limit with respect to a Euclidean integrand [DM, Theorem 20.3], which applies to a large class of integral functionals. Let $c_{1}, c_{2}, c_{3}$ be real numbers with $c_{i} \geq 0 i=1,2,3$. Let us denote by $\mathcal{H}=\mathcal{H}\left(p, c_{1}, c_{2}, c_{3}\right)$ the class of all local functionals $F: L^{p}(\Omega) \times \mathcal{A} \rightarrow[0, \infty]$ for which there exist two Borel functions $f_{e}, g: \Omega \times \mathbb{R}^{n} \rightarrow[0, \infty)$ (depending on $F$ ) such that

(a) $F(u, A):=\left\{\begin{array}{ll}\int_{A} f_{e}(x, D u) d x & \text { if } A \in \mathcal{A}, u \in W_{\mathrm{loc}}^{1,1}(A) \\ \infty & \text { otherwise }\end{array}\right.$;

(b) $g(x, \xi) \leq f_{e}(x, \xi) \leq c_{1}(g(x, \xi)+1)$;

(c) $0 \leq g(x, \xi) \leq c_{2}\left(|\xi|^{p}+1\right)$;

(d) $g(x, \cdot)$ is convex on $\mathbb{R}^{n}$;

(e) $g(x, 2 \xi) \leq c_{3}(g(x, \xi)+1)$, 
for every $u \in L^{p}(\Omega), x \in \Omega, \xi \in \mathbb{R}^{n}$.

4.12. Theorem. For every sequence $\left(F_{h}\right)_{h}$ of functionals of the class $\mathcal{H}$ there exist a subsequence $\left(F_{h_{k}}\right)_{k}$ and an increasing functional $F: L^{p}(\Omega) \times$ $\mathcal{A} \rightarrow[0, \infty]$ such that $\left(F_{h_{k}}\right)_{k} \bar{\Gamma}$-converges to $F$. The functional $F$ can be represented in integral form by a Euclidean integrand, that is, there exists a Borel function $f_{e}: \Omega \times \mathbb{R}^{n} \rightarrow[0, \infty]$ verifying

(i) $f_{e}(x, \cdot)$ is convex on $\mathbb{R}^{n}$;

(ii) $0 \leq f_{e}(x, \xi) \leq c_{1}\left(c_{2}+1\right)+c_{1} c_{2}|\xi|^{p}$ for a.e. $x \in \Omega$, for each $\xi \in \mathbb{R}^{n}$, such that (10) holds.

Let us also recall an useful criterion for proving that a class of local functionals on $L^{p}(\Omega) \times \mathcal{A}$ satisfies the fundamental estimate uniformly [DM, Theorem 19.4] and a $\bar{\Gamma}$-compacness result in this class [DM, Theorem 19.5].

4.13. Theorem. Let $c_{i}(i=1,2,3,4)$ be non negative real numbers and let $\sigma: \mathcal{A} \rightarrow[0, \infty]$ be a superadditive increasing set function such that $\sigma(A)<\infty$ for each $A \Subset \Omega$. Let $\mathcal{F}^{\prime}=\mathcal{F}^{\prime}\left(p, c_{1}, c_{2}, c_{3}, c_{4}\right)$ be the class of all non-negative increasing local functionals $F: L^{p}(\Omega) \times \mathcal{A} \rightarrow[0, \infty]$ with the following properties: $F$ is a measure and there exists a non-negative increasing local functional $G: L^{p}(\Omega) \times \mathcal{A} \rightarrow[0, \infty]$ (depending on $F$ ) such that $G$ is a measure and

$$
\begin{aligned}
& G(u, A) \leq F(u, A) \leq c_{1} G(u, A)+c_{2}\|u\|_{L^{p}(A)}^{p}+\sigma(A) ; \\
& G(\varphi u+(1-\varphi) v, A) \leq c_{4}(G(u, A)+G(v, A))+ \\
& \quad+c_{3} c_{4} \max _{\Omega}|D \varphi|^{p}\|u-v\|_{L^{p}(A)}^{p}+2 c_{4} \sigma(A),
\end{aligned}
$$

for every $u, v \in L^{p}(\Omega), A \in \mathcal{A}, \varphi \in \mathbf{C}_{c}^{\infty}(\Omega)$ with $0 \leq \varphi \leq 1$. Then, the fundamental estimate holds uniformly on $\mathcal{F}^{\prime}$.

4.14. Theorem. Let $\mathcal{F}^{\prime}=\mathcal{F}^{\prime}\left(p, c_{1}, c_{2}, c_{3}, c_{4}\right)$ be the class of local functionals defined in Theorem 4.13. For every sequence $\left(F_{h}\right)_{h} \subset \mathcal{F}^{\prime}$, there exists a subsequence $\left(F_{h_{k}}\right)_{k}$ which $\bar{\Gamma}$-converges to a lower semicontinuous functional $F \in \mathcal{F}^{\prime}$.

Let us now introduce some results concerning functionals depending on vector fields. Let us first prove a $\Gamma$-compactness result (see Theorem4.16) for a class of local functional on $L^{p}(\Omega) \times \mathcal{A}$ satisfying suitable growth conditions with respect to the local functional $\Psi_{p}: L^{p}(\Omega) \times \mathcal{A} \rightarrow[0, \infty]$ defined as

$$
\Psi_{p}(u, A):=\left\{\begin{array}{ll}
\int_{A}|X u|^{p} d x & \text { if } A \in \mathcal{A}, u \in W_{X}^{1, p}(A) \\
+\infty & \text { otherwise }
\end{array} .\right.
$$

As a consequence, we will get a $\Gamma$-compactness result for a class of integral functionals represented with respect to Euclidean integrands, but still with growth condition with respect to to $\Psi_{p}$ (see Theorem 4.17). The former is an extension of [DM, Theorem 19.6], the latter of [DM, Theorem 20.4]. 
4.15. Lemma. Let $p>1$. Then $\Psi_{p}: L^{p}(\Omega) \times \mathcal{A} \rightarrow[0, \infty]$ is a measure and lsc.

Proof. Let us start by proving that for any $A \in \mathcal{A}$ the function $u \rightarrow \Psi_{p}(u, A)$ is $L^{p}$-lsc, i.e., for any $A \in \mathcal{A}$ and $\left(u_{h}\right)_{h} \subset L^{p}(\Omega), u_{h} \rightarrow u$ in $L^{p}(\Omega)$, it satisfies

$$
\Psi_{p}(u, A) \leq \liminf _{h \rightarrow \infty} \Psi_{p}\left(u_{h}, A\right) .
$$

We can assume $\liminf \operatorname{in}_{h \rightarrow \infty} \Psi_{p}\left(u_{h}, A\right)<\infty$. Therefore, up to a subsequence, we can also assume that $\lim _{h \rightarrow \infty} \Psi_{p}\left(u_{h}, A\right)$ exists. Hence $\left(u_{h}\right)_{h}$ is bounded in $W_{X}^{1, p}(A)$ and, since $W_{X}^{1, p}(A)$ is reflexive (recall Proposition 2.5 and that $p>$ 1 ), we get a subsequence $u_{h} \rightarrow u$ in $W_{X}^{1, p}(A)$ and, in particular, $X u_{h} \rightarrow X u$ in $L^{p}(A)$, which implies the conclusion, recalling the lower semicontinuity of the $L^{p}$-norm with respect to the weak convergence.

We now prove that for any $u \in L^{p}(\Omega)$ the function $\Psi_{p}(u, \cdot): \mathcal{A} \rightarrow[0, \infty]$ is a measure, i.e., there exists a Borel measure $\mu_{u}: \mathcal{B}(\Omega) \rightarrow[0, \infty]$ such that $\Psi_{p}(u, A)=\mu_{u}(A)$ for every $A \in \mathcal{A}$. Since, by Remark 3.10, $\Psi_{p}(u, \cdot)$ is nonnegative, increasing and such that $\Psi_{p}(u, \emptyset)=0$, it suffices to prove that $\Psi_{p}(u, \cdot)$ is subadditive, superadditive and inner regular.

$\Psi_{p}(u, \cdot)$ is subadditive, namely for every $A, A_{1}, A_{2} \in \mathcal{A}$ with $A \subseteq A_{1} \cup A_{2}$

$$
\Psi_{p}(u, A) \leq \Psi_{p}\left(u, A_{1}\right)+\Psi_{p}\left(u, A_{2}\right) .
$$

We can assume $u \in W_{X}^{1, p}\left(A_{1}\right) \cap W_{X}^{1, p}\left(A_{2}\right)$ and $A_{1}, A_{2} \in \mathcal{A}$, otherwise the conclusion is trivial. Remark 2.6 (ii) gives $u \in W_{X}^{1, p}\left(A_{1} \cup A_{2}\right)$, therefore $\Psi_{p}\left(u, A_{1} \cup A_{2}\right)=\int_{A_{1} \cup A_{2}}|X u|^{p} d x$ and (83) follows.

$\Psi_{p}(u, \cdot)$ is superadditive, namely for every $A, A_{1}, A_{2} \in \mathcal{A}$ with $A_{1} \cup A_{2} \subseteq A$ and $A_{1} \cap A_{2}=\emptyset$

$$
\Psi_{p}(u, A) \geq \Psi_{p}\left(u, A_{1}\right)+\Psi_{p}\left(u, A_{2}\right) .
$$

We can assume $u \in W_{X}^{1, p}(A)$ and $A \in \mathcal{A}$, otherwise the conclusion is trivial. Remark 2.6 (iv) gives $u \in W_{X}^{1, p}(B)$ for any open set $B \subseteq A$. Let $A, A_{1}, A_{2} \in$ $\mathcal{A}, A_{1} \cup A_{2} \subseteq A$ and $A_{1} \cap A_{2}=\emptyset$. Then

$$
\Psi_{p}\left(u, A_{1}\right)+\Psi_{p}\left(u, A_{2}\right)=\int_{A_{1} \cup A_{2}}|X u|^{p} d x \leq \int_{A}|X u|^{p} d x
$$

and (84) follows.

$\Psi_{p}(u, \cdot)$ is inner regular, namely for every $A \in \mathcal{A}$

$$
\Psi_{p}(u, A)=\sup \left\{\Psi_{p}(u, B) \mid B \in \mathcal{A}, B \Subset A\right\} .
$$

Let $M:=\sup \left\{\Psi_{p}(u, B) \mid B \in \mathcal{A}, B \Subset A\right\} \in[0,+\infty]$. If $M=+\infty$, there exists $\left\{B_{i}\right\}_{i \in \mathbb{N}} \subset \mathcal{A}, B_{i} \Subset A$ such that $\Psi_{p}\left(u, B_{i}\right) \rightarrow \infty$ as $i \rightarrow+\infty$ and the conclusion follows by observing that for all $i \in \mathbb{N}, \Psi_{p}\left(u, B_{i}\right) \leq \Psi_{p}(u, A)$. If $M \in[0, \infty)$, then $\|u\|_{W_{X}^{1, p}(B)} \leq M$ for any $B \in \mathcal{A}, B \Subset A$. Then, Remark (2.6) (iii) gives $u \in W_{X}^{1, p}(A)$ and, by definition, $\Psi_{p}(u, A)=\int_{A}|X u|^{p} d x$. For 
any $\varepsilon>0$ there exists $\delta>0$ such that $\int_{E}|X u|^{p} d x \leq \varepsilon$ for any $E \in \mathcal{A}$ with $|E| \leq \delta$. Let $B \Subset A$ such that $|A \backslash \bar{B}| \leq \delta$, then

$$
\int_{A}|X u|^{p} d x=\int_{B}|X u| d x+\int_{A \backslash \bar{B}}|X u|^{p} d x \leq \int_{B}|X u|^{p} d x+\varepsilon
$$

and the thesis follows.

4.16. Theorem. Let $p>1, \Omega \subset \mathbb{R}^{n}$ be a bounded open set and $c_{1} \geq c_{0}>0$. Denote by $\mathcal{M}=\mathcal{M}\left(p, c_{0}, c_{1}\right)$ the class of local functionals $F: L^{p}(\Omega) \times \mathcal{A} \rightarrow$ $[0, \infty]$ such that $F$ is a measure and

$$
c_{0} \Psi_{p}(u, A) \leq F(u, A) \leq c_{1}\left(\Psi_{p}(u, A)+\|u\|_{L^{p}(A)}^{p}+|A|\right)
$$

for every $u \in L^{p}(\Omega)$ and for every $A \in \mathcal{A}$. Then, the fundamental estimate holds uniformly in $\mathcal{M}$ and every sequence $\left(F_{h}\right)_{h} \subset \mathcal{M}$ has a subsequence $\left(F_{h_{k}}\right)_{k}$ which $\bar{\Gamma}$-converges to a functional $F$ of the class $\mathcal{M}$. Moreover, $\left(F_{h_{k}}(\cdot, A)\right)_{k} \Gamma$-converges to $F(\cdot, A)$ in $L^{p}(\Omega)$ and

$$
\operatorname{dom} F(\cdot, A):=\left\{u \in L^{p}(\Omega): F(u, A)<\infty\right\}=W_{X}^{1, p}(A)
$$

for every $A \in \mathcal{A}$.

Proof. Let us begin to prove that the fundamental estimate holds uniformly in $\mathcal{M}$. Let

$$
g(x, \xi):=c_{0}|C(x) \xi|^{p} \quad \text { if } x \in \Omega, \xi \in \mathbb{R}^{n} .
$$

Notice that, since the entries of matrix $C(x)$ are Lipschitz continuous functions,

$$
\begin{array}{ll}
g(x, \xi) \leq c_{0} \sup _{\Omega}\|C(x)\|^{p}|\xi|^{p}=c_{2}|\xi|^{p} & \text { if } x \in \Omega, \xi \in \mathbb{R}^{n}, \\
g(x, 2 \xi)=2^{p-1} 2 g(x, \xi)=c_{3} 2 g(x, \xi) & \text { if } x \in \Omega, \xi \in \mathbb{R}^{n}
\end{array}
$$

and

$$
g(x, \cdot) \text { is convex on } \mathbb{R}^{n} .
$$

Thus, from (89), (90) and (91), arguing as in [DM, (19.6)], it follows that

$$
g(x, t \xi+(1-t) \eta+\zeta) \leq c_{3}(g(x, \xi)+g(x, \eta))+c_{2}|\zeta|^{p}
$$

for every $x \in \Omega, t \in[0,1], \xi, \eta \in \mathbb{R}^{n}$. We are going to apply Theorem 4.13, Observe that, choosing $G=c_{0} \Psi_{p}$, from (86), (799) immediately holds with

$$
c_{1} \equiv \frac{c_{1}}{c_{0}}, c_{2} \equiv c_{1}, \sigma(A)=c_{1}|A|
$$


Let us show (80). By (92), it follows that

$$
\begin{aligned}
& G(\varphi u+(1-\varphi) v, A)=\int_{A} g(x, \varphi D u+(1-\varphi) D v+(u-v) D \varphi) d x \\
& \leq \int_{A}\left[c_{3}\left(g(x, D u)+g(x, D v)+c_{2}|D \varphi|^{p}|u-v|^{p}\right] d x\right. \\
& \leq c_{3}(G(u, A)+G(v, A))+c_{2}\left(\max _{\Omega}|D \varphi|^{p}\right)\|u-v\|_{L^{p}(A)}^{p}
\end{aligned}
$$

for each $u, v \in L^{p}(\Omega), A \in \mathcal{A}, \varphi \in \mathbf{C}_{c}^{\infty}(\Omega)$ with $0 \leq \varphi \leq 1$. Thus (80) holds with

$$
c_{4} \equiv c_{3} \text { and } c_{3} c_{4} \equiv c_{2} .
$$

Thus we get the desired conclusion. From Theorem 4.14, every sequence $\left(F_{h}\right)_{h} \subset \mathcal{M}$ has a subsequence $\left(F_{h_{k}}\right)_{k} \bar{\Gamma}$-converging to a functional $F$ : $L^{p}(\Omega) \times \mathcal{A} \rightarrow[0, \infty]$ which is a measure. As each functional $F_{h}$ satisfies (86)), the functional $F$ satisfies (86), since $\Psi_{p}$ is lsc and inner regular by Lemma 4.15 and Remark 3.10, By applying Theorem 4.8, we get that $\left(F_{h_{k}}(\cdot, A)\right)_{k}$ $\Gamma$-converges to $F(\cdot, A)$ in $L^{p}(\Omega)$ for each $A \in \mathcal{A}$, since $\Omega$ is bounded. Finally, by (86), (87) follows.

Let $p>1$ and let $c_{1} \geq c_{0}$, let $\Omega \subset \mathbb{R}^{n}$ be a bounded open set. Let us denote by $\mathcal{I}=\mathcal{I}\left(p, c_{0} . c_{1}\right)$ the class of local functionals $F: L^{p}(\Omega) \times \mathcal{A} \rightarrow$ $[0, \infty]$ for which there exists a Borel function $f_{e}: \Omega \times \mathbb{R}^{n} \rightarrow[0, \infty)$ such that

(i) claim (a) of properties defining $\mathcal{H}$ holds;

(ii) $c_{0}|C(x) \xi|^{p} \leq f_{e}(x, \xi) \leq c_{1}\left(|C(x) \xi|^{p}+1\right)$ a.e. $x \in \Omega$, for each $\xi \in$ $\mathbb{R}^{n}$.

4.17. Theorem. For every sequence $\left(F_{h}\right)_{h} \subset \mathcal{I}$ there exist a subsequence $\left(F_{h_{k}}\right)_{k}$ and a measure functional $F: L^{p}(\Omega) \times \mathcal{A} \rightarrow[0, \infty]$ such that $\left(F_{h_{k}}(\cdot, A)\right)_{k}$ $\Gamma$-converges to $F(\cdot, A)$ in $L^{p}(\Omega)$ and (87) holds for every $A \in \mathcal{A}$. Moreover there exists a Borel function $f_{e}: \Omega \times \mathbb{R}^{n} \rightarrow[0, \infty)$, convex in the second variable and satisfying (ii), for which (10) holds.

Proof. By Theorem 4.16, for each $\left(F_{h}\right)_{h} \subset \mathcal{I}$ there exist a subsequence $\left(F_{h_{k}}\right)_{k}$ and an inner regular functional $F: L^{p}(\Omega) \times \mathcal{A} \rightarrow[0, \infty]$ such that $\left(F_{h_{k}}(\cdot, A)\right)_{k} \Gamma$-converges to $F(\cdot, A)$ in $L^{p}(\Omega)$ for every $A \in \mathcal{A}$. Moreover, since $\Psi_{p}$ is lsc and inner regular, for each $u \in L^{p}(\Omega), A \in \mathcal{A}$,

$$
c_{0} \Psi_{p}(u, A) \leq F(u, A) \leq c_{1}\left(\Psi_{p}(u, A)+|A|\right)
$$

where $\Psi_{p}$ is the local functional in (81). If $g(x, \xi)$ is as in (88), $\mathcal{I}\left(p, c_{0} . c_{1}\right) \subset$ $\mathcal{H}\left(p, c_{1}^{\prime}, c_{2}^{\prime}, c_{3}^{\prime}\right)$, for suitable $c_{i}^{\prime}(i=1,2,3)$. From Theorem 4.12 , there exists a Borel function $f_{e}: \Omega \times \mathbb{R}^{n} \rightarrow[0, \infty)$, also convex in the second variable, for which (10) holds.

Let us now prove that (ii) of properties defining $\mathcal{I}$ holds. Let $u_{\xi}$ be the function in (49). From (93), it follows that

$$
c_{0} \int_{A}|C(x) \xi|^{p} d x \leq \int_{A} f_{e}(x, \xi) d x \leq c_{1}\left(|A|+\int_{A}|C(x) \xi|^{p} d x\right)
$$


for each $\xi \in \mathbb{R}^{n}$ and $A \in \mathcal{A}$. By means of the usual procedure, we can infer that there exists a negligeble set $\mathcal{N} \subset \Omega$ such that, for each $x \in \Omega \backslash \mathcal{N}$,

$$
c_{0}|C(x) \xi|^{p} \leq f_{e}(x, \xi) \leq c_{1}\left(|C(x) \xi|^{p}+1\right) \quad \forall \xi \in \mathbb{Q}^{n} .
$$

Then, since $f_{e}(x, \cdot): \mathbb{R}^{n} \rightarrow[0, \infty)$ is continuous a.e. $x \in \Omega$, we can extend the previous inequality to all $\xi \in \mathbb{R}^{n}$.

4.18. Theorem. Let $\Omega \subset \mathbb{R}^{n}$ be a bounded open set, let $\left(f_{h}\right)_{h} \subset I_{m . p}\left(\Omega, c_{0}, c_{1}\right)$ and, for each $h$, let $F_{h}^{*}: L^{p}(\Omega) \times \mathcal{A} \rightarrow[0, \infty]$ be the local functional defined in (77). Then, there exist a subsequence $\left(F_{h_{k}}^{*}\right)_{k}$ and a measure functional $F: L^{p}(\Omega) \times \mathcal{A} \rightarrow[0, \infty]$ such that $\left(F_{h_{k}}^{*}(\cdot, A)\right)_{k} \Gamma$-converges to $F(\cdot, A)$ in $L^{p}(\Omega)$ and (87) holds for every $A \in \mathcal{A}$. Moreover, there exists a Borel function $f_{e}: \Omega \times \mathbb{R}^{n} \rightarrow[0, \infty)$, convex in the second variable, satisfying (ii) of properties defining $\mathcal{I}$, for which (10) holds.

Proof. Let $\left(f_{h, e}\right)_{h}$ denote the sequence of Euclidean integrands in (8) and let $\left(F_{h}\right)_{h}$ be the sequence of local functionals in (7). Since $\left(f_{h, e}\right)_{h} \subset \mathcal{I}$, by applying Theorem 4.17 there exist a subsequence $\left(F_{h_{k}}\right)_{k}$ and a measure functional $F: L^{p}(\Omega) \times \mathcal{A} \rightarrow[0, \infty]$ such that $\left(F_{h_{k}}(\cdot, A)\right)_{k} \Gamma$-converges to $F(\cdot, A)$ in $L^{p}(\Omega)$ for every $A \in \mathcal{A}$. Moreover, there exists a Borel function $f_{e}: \Omega \times \mathbb{R}^{n} \rightarrow[0, \infty)$, convex in the second variable, satisfying (ii), for which (10) holds.

By Theorem 3.1 (iii), it follows that, for each $h \in \mathbb{N}, A \in \mathcal{A}$,

$$
F_{h}^{*}(\cdot, A)=\bar{F}_{h}(\cdot, A)
$$

where $\bar{F}_{h}(\cdot, A): L^{p}(\Omega) \rightarrow[0, \infty]$ denotes the relaxed functional of $F_{h}(\cdot, A)$ : $L^{p}(\Omega) \rightarrow[0, \infty]$ with respect to the $L^{p}(\Omega)$ topology (see (22)). By (94) and a well-known property of $\Gamma$-convergence (see [DM, Propostion 6.11]), we also get that $\left(F_{h_{k}}^{*}(\cdot, A)\right)_{k} \Gamma$-converges to $F(\cdot, A)$ in $L^{p}(\Omega)$ for every $A \in \mathcal{A}$.

4.19. Theorem. Let $\Omega \subset \mathbb{R}^{n}$ be a bounded open set and let $X=\left(X_{1}, \ldots, X_{m}\right)$ satisfy (LIC) on $\Omega$. Let $\left(f_{h}\right)_{h} \subset I_{m, p}\left(\Omega, c_{0}, c_{1}\right)$ and, for each $h$, let $F_{h}^{*}$ : $L^{p}(\Omega) \times \mathcal{A} \rightarrow[0, \infty]$ be the local functional defined in (77). Assume that:

(i) there exists a measure functional $F: L^{p}(\Omega) \times \mathcal{A} \rightarrow[0, \infty]$ such that $\left(F_{h}^{*}\right)_{h} \Gamma$-converges to $F(\cdot, A)$ in $L^{p}(\Omega)$ and (87) holds for each $A \in \mathcal{A}$;

(ii) there exists a Borel function $f_{e}: \Omega \times \mathbb{R}^{n} \rightarrow[0, \infty)$, convex in the second variable, satisfying (ii) of properties defining $\mathcal{I}$, for which $F$ admits the integral representation in (10).

(iii) (87) holds for every $A \in \mathcal{A}$.

Then, there exists $f \in I_{m, p}\left(\Omega, c_{0}, c_{1}\right)$ for which $F$ admits the integral representation (78).

Proof. Let us first notice that $f_{e}$ satisfies the assumptions of Lemma 3.14. Thus we can assume that it satisfies (47). 
Let $f: \Omega \times \mathbb{R}^{m} \rightarrow[0, \infty]$ be the function in (44). Let us prove that $f \in I_{m, p}\left(\Omega, c_{0}, c_{1}\right)$. Properties $\left(I_{1}\right)$ and $\left(I_{2}\right)$ follow from Therem 3.5. Since $f_{e}$ satisfies (ii) of properties defining class $\mathcal{I}$, from (100), we can infer $\left(I_{3}\right)$.

From Theorem 3.5 and Remark 3.8, $F$ admits the integral representation (78), but only for functions $u \in W^{1, p}(A)$. We are going to extend this representation to all functions $u \in W_{X}^{1, p}(A)$, by means of Theorem 3.12 about the integral representation of local functionals with respect to $X$ gradient. Being $F$ a $\Gamma$-limit, it is lsc (see [DM, Proposition 6.8]) and, by [DM, Proposition 16.15], it is also local and, by assumptions, a measure. Thus assumptions (a), (b) and (c) of Theorem 3.12 are satisfied. Let us prove assumtion (d). For every $h \in \mathbb{N}$, we have $F_{h}^{*}(u+c, A)=F_{h}^{*}(u, A)$ whenever $u \in L^{p}(\Omega), c \in \mathbb{R}$. Then it is easy to see that this property also holds for the $\Gamma$-limit $F$. Let us now prove assumption (e). By the integral representation (10) and Remark 3.8, it follows that, for each $A \in \mathcal{A}$, $u \in W^{1, p}(A)$

$$
\begin{aligned}
F(u, A) & =\int_{A} f_{e}(x, D u) d x=\int_{A} f(x, X u) d x \\
& \leq c_{1}\left(\int_{A}|X u|^{p}+|A|\right)
\end{aligned}
$$

which implies property (e). Thus there exists a Borel function $f^{*}: \Omega \times \mathbb{R}^{m} \rightarrow$ $[0, \infty]$ satisfying property (i) and (ii) of Theorem 3.12. In particular, for each $A \in \mathcal{A}, u \in W_{X}^{1, p}(A)$

$$
F(u, A)=\int_{A} f^{*}(x, X u) d x .
$$

By (95) and Theorem 3.5, we get that $f(x, \eta)=f^{*}(x, \eta)$ for a.e. $x \in \Omega$ and for each $\eta \in \mathbb{R}^{m}$. This concludes the proof.

Proof of Theorem 4.11. The proof immediately follows from Theorems 4.18 and 4.19 .

We now introduce two integrand function subclasses $J_{i} \subset I_{m, p}\left(\Omega, c_{0}, c_{1}\right)$ $(i=1,2)$ for which the associated functionals in (5) are still compact with respect to $\Gamma$ - convergence in $L^{p}(\Omega)$-topology. Let $\Omega \subset \mathbb{R}^{n}$ be a bounded open set and let us fix $0<c_{0} \leq c_{1}$.

- $J_{1} \equiv J_{1}\left(\Omega, c_{0}, c_{1}\right)$ is the subclass of $I_{m, 2}\left(\Omega, c_{0}, c_{1}\right)$ composed of integrand functions $f \in I_{m, 2}\left(\Omega, c_{0}, c_{1}\right)$ which are quadratic forms with respect to $\eta$, that is,

$f(x, \eta)=\langle a(x) \eta, \eta\rangle=\sum_{i, j=1}^{m} a_{i j}(x) \eta_{i} \eta_{j} \quad$ a.e. $x \in \Omega, \forall \eta \in \mathbb{R}^{m}$,

with $a(x)=\left[a_{i j}(x)\right] m \times m$ symmetric matrix . 
- The subclass $J_{2} \equiv J_{2}\left(\Omega, c_{0}, c_{1}\right)$ is composed by integrand functions $f \in I_{m, p}\left(\Omega, c_{0}, c_{1}\right)$ such that $f=f(\eta)$, that is, $f$ is independent of $x$.

4.20. Theorem. Let $\Omega \subset \mathbb{R}^{n}$ be a bounded open set and let $X=\left(X_{1}, \ldots, X_{m}\right)$ satisfy (LIC) on $\Omega$. Let $\left(f_{h}\right)_{h} \subset J_{i}\left(\Omega, c_{0}, c_{1}\right)(i=1,2)$ and, for each $h$, let $F_{h}^{*}: L^{p}(\Omega) \times \mathcal{A} \rightarrow[0, \infty]$ be the local functional defined in (77). Then, up to a subsequence, there exist a local functional $F: L^{p}(\Omega) \times \mathcal{A} \rightarrow[0, \infty]$ and $f \in J_{i}\left(\Omega, c_{0}, c_{1}\right)$ such that

(i) (9) holds;

(ii) $F$ admits representation (178).

Proof. 1st case. Let us first show the conclusion for the subclass $J_{1}$.

Let $\left(f_{h}\right)_{h} \subset J_{1}$. By definition, we can assume that

$$
f_{h}(x, \eta):=\left\langle a_{h}(x) \eta, \eta\right\rangle \quad x \in \Omega, \eta \in \mathbb{R}^{m},
$$

where $a_{h}(x)=\left[a_{h, i j}(x)\right]$ is a $m \times m$ symmetric matrix satisfying

$$
\begin{gathered}
c_{0}|\eta|^{2} \leq\left\langle a_{h}(x) \eta, \eta\right\rangle \leq c_{1}\left(|\eta|^{2}+1\right) \text { a.e. } x \in \Omega, \forall \eta \in \mathbb{R}^{m} \\
a_{h, i j} \in L^{\infty}(\Omega) \text { for each } i, j=1, \ldots, m, h \in \mathbb{N} .
\end{gathered}
$$

Applying Theorem 4.11, up to a subsequence, there exist a local functional $F: L^{p}(\Omega) \times \mathcal{A} \rightarrow[0, \infty]$ and $f \in I_{m, 2}\left(\Omega, c_{0}, c_{1}\right)$ such that (9) holds and $F$ admits representation (78). We have only to prove that

$$
f \in J_{1} \text {. }
$$

Notice that we can also assume that $F$ admits representation (10) with

$$
f_{e}(x, \xi):=f(x, C(x) \xi) \text { for a.e. } x \in \Omega \text {, for each } \xi \in \mathbb{R}^{n} .
$$

Moreover, by Theorem 3.5 (see (44) and (40)), it also holds the opposite representation, that is, for each $x \in \Omega_{X}$,

$$
f(x, \eta)=f_{e}\left(x, L_{x}^{-1}(\eta)\right) \quad \forall \eta \in \mathbb{R}^{m},
$$

with

$$
L_{x}^{-1}(\eta):=C(x)^{T} B(x)^{-1} \eta .
$$

Let us now consider the sequence of Euclidean integrands

$$
\begin{aligned}
f_{h, e}(x, \xi) & :=f_{h}(x, C(x) \xi)=\left\langle a_{h}(x) C(x) \xi, C(x) \xi\right\rangle \\
& =\left\langle C(x)^{T} a_{h}(x) C(x) \xi, \xi\right\rangle=\left\langle a_{h, e}(x) \xi, \xi\right\rangle
\end{aligned}
$$

and the related local functionals $F_{h}: L^{p}(\Omega) \times \mathcal{A} \rightarrow[0, \infty]$ defined in (7). Since $F_{h}(u, A)=F_{h}^{*}(u, A)$ for each $u \in W_{\text {loc }}^{1,1}(A)$, by using well-known results of $\Gamma$-convergence for quadratic functionals (see [DM, Theorem 22.1] and Remark 4.5. one can easily prove that there exists a $n \times n$ symmetric matrix $a_{e}(x)=\left[a_{e, i j}(x)\right]$, with $a_{e, i j} \in L^{\infty}(\Omega)$ for each $i, j=1, \ldots, n$ such that

$$
f_{e}(x, \xi)=\left\langle a_{e}(x) \xi, \xi\right\rangle \text { a.e. } x \in \Omega, \forall \xi \in \mathbb{R}^{n} \text {. }
$$


By (99), for each $x \in \Omega_{X}$,

$$
\begin{aligned}
f(x, \eta) & :=f_{e}\left(x, L_{x}^{-1}(\eta)\right)=\left\langle a_{e}(x) C(x)^{T} B(x)^{-1} \eta, C(x)^{T} B(x)^{-1} \eta\right\rangle \\
& =\left\langle\left(B(x)^{-1}\right)^{T} C(x) a_{e}(x) C(x)^{T} B(x)^{-1} \eta, \eta\right\rangle=\langle a(x) \eta, \eta\rangle
\end{aligned}
$$

with

$$
a(x):=\left(B(x)^{-1}\right)^{T} C(x) a_{e}(x) C(x)^{T} B(x)^{-1},
$$

$m \times m$ symmetric matrix. Then $f(x, \cdot)$ turns out to be a quadratic form on $\mathbb{R}^{m}$, induced by the matrix $a(x)$ for a.e. $x \in \Omega$. Thus (98) follows.

2nd case. Let us now deal with the subclass $J_{2}$. Let $\left(f_{h}\right)_{h} \subset J_{2}$. Notice that $f_{h}: \mathbb{R}^{m} \rightarrow[0, \infty), h \in \mathbb{N}$, is a sequence of locally bounded, convex functions. Thus, by a well-known result (see, for instance, DM, Proposition 5.11]), we can infer that $\left(f_{h}\right)_{h}$ is also locally equi-Lipschitz continuous. From Ascoli-Arzelà' s theorem, we can assume that, up to a subsequence, there exists $f \in J_{2}$ such that

$$
f_{h} \rightarrow f \text { uniformly on bounded sets of } \mathbb{R}^{n} \text { as } h \rightarrow \infty \text {. }
$$

Let us define $\tilde{F}: L^{p}(\Omega) \times \mathcal{A} \rightarrow[0, \infty]$ as

$$
\tilde{F}(u, A):=\left\{\begin{array}{ll}
\int_{A} f(X u(x)) d x & \text { if } A \in \mathcal{A}, u \in W_{X}^{1, p}(A) \\
\infty & \text { otherwise }
\end{array} .\right.
$$

Let us now prove that, for each $A \in \mathcal{A}$,

$$
\lim _{h \rightarrow \infty} F_{h}^{*}(u, A)=\tilde{F}(u, A) \quad \forall u \in W_{X}^{1, p}(A) .
$$

Let us fix $A \in \mathcal{A}$ and $u \in W_{X}^{1, p}(A)$. Since $|X u(x)|<\infty$ for a.e. $x \in A$, by (101), it follows that

$$
\lim _{h \rightarrow \infty} f_{h}(X u(x))=f(X u(x)) \text { for a.e. } x \in A .
$$

On the other hand, as

$$
0 \leq f_{h}(X u(x)) \leq c_{1}\left(1+|X u(x)|^{p}\right) \text { for a.e. } x \in A \text {, for each } h,
$$

by (103) and the dominated convergence theorem, (102) follows. We have only to prove that

$$
F(u, A)=\tilde{F}(u, A) \quad \forall A \in \mathcal{A}, \forall u \in L^{p}(\Omega)
$$

in order to get our desired conclusion. By (87), it is sufficient to prove (104) for each $A \in \mathcal{A}$ and for each $u \in W_{X}^{1, p}(A)$. The inequality

$$
F(u, A) \leq \tilde{F}(u, A) \quad \forall A \in \mathcal{A}, \forall u \in W_{X}^{1, p}(A),
$$

follows by noticing that, for each $u \in W_{X}^{1, p}(A)$, by $\Gamma-\lim$ inf inequality and (102)

$$
F(u, A) \leq \liminf _{h \rightarrow \infty} F_{h}^{*}(u, A)=\tilde{F}(u, A) .
$$

Let us now prove the opposite inequality

$$
F(u, A) \geq \tilde{F}(u, A) \quad \forall A \in \mathcal{A}, \forall u \in W_{X}^{1, p}(A) .
$$


Let us first recall that, for each $A \in \mathcal{A}$, by (102) and Proposition 4.9,

$$
\tilde{F}(u, A)=\left(\Gamma\left(W_{X}^{1, p}(A)\right)-\lim _{h \rightarrow \infty} F_{h}^{*}\right)(u) \quad \forall u \in W_{X}^{1, p}(A) .
$$

Fix $A \in \mathcal{A}$ and let $u \in L^{p}(\Omega)$ with $\left.u\right|_{A} \in W_{X}^{1, p}(A)$. By the $\Gamma-\lim$ equality, there exists a sequence $\left(u_{h}\right)_{h} \subset L^{p}(\Omega)$ such that

$$
u_{h} \rightarrow u \text { in } L^{p}(\Omega) \text {, as } h \rightarrow \infty
$$

and

$$
\lim _{h \rightarrow \infty} F_{h}^{*}\left(u_{h}, A\right)=F(u, A)<\infty .
$$

By (109), we can assume that

$$
\left(\left.u_{h}\right|_{A}\right)_{h} \subset W_{X}^{1, p}(A) .
$$

Let $A^{\prime} \in \mathcal{A}$ with $A^{\prime} \Subset A$. From Proposition 2.10 (ii), if $w:=\overline{X u_{h}}$ : $\mathbb{R}^{n} \rightarrow \mathbb{R}^{m}$, that is, $\overline{X u_{h}}=X u_{h}$ on $A$ and $\overline{X u_{h}}=0$ outside, for each $0<\varepsilon<\operatorname{dist}\left(A^{\prime}, \mathbb{R}^{n} \backslash A\right)$

$$
\int_{A^{\prime}} f_{h}\left(\rho_{\epsilon} * \overline{X u_{h}}\right) d x \leq \int_{A} f_{h}\left(\overline{X u_{h}}\right) d x \text { for each } h .
$$

By (108), (110) and Proposition 2.10 (i), for given $0<\varepsilon<\operatorname{dist}\left(A^{\prime}, \mathbb{R}^{n} \backslash A\right)$,

$$
X\left(\rho_{\epsilon} * \bar{u}_{h}\right) \rightarrow X\left(\rho_{\epsilon} * \bar{u}\right) \text { uniformly on } A^{\prime} \text { as } h \rightarrow \infty
$$

and

$$
\rho_{\epsilon} * \overline{X u_{h}} \rightarrow \rho_{\epsilon} * \overline{X u} \text { uniformly on } A^{\prime} \text { as } h \rightarrow \infty .
$$

In particular,

$$
\rho_{\varepsilon} * \bar{u}_{h} \rightarrow \rho_{\varepsilon} * \bar{u} \text { in } W_{X}^{1, p}\left(A^{\prime}\right) \text { as } h \rightarrow \infty .
$$

Observe now that, by (111), for each $0<\varepsilon<\operatorname{dist}\left(A^{\prime}, \mathbb{R}^{n} \backslash A\right)$, for each $h$,

$$
\begin{aligned}
& F_{h}^{*}\left(\rho_{\epsilon} * \bar{u}_{h}, A^{\prime}\right)=\int_{A^{\prime}} f_{h}\left(X\left(\rho_{\epsilon} * \bar{u}_{h}\right)\right) d x \\
& =\int_{A^{\prime}} f_{h}\left(\rho_{\epsilon} * \overline{X u_{h}}\right) d x+\int_{A^{\prime}}\left(f_{h}\left(X\left(\rho_{\epsilon} * \bar{u}_{h}\right)\right)-f_{h}\left(\rho_{\epsilon} * \overline{X u_{h}}\right)\right) d x \\
& \leq \int_{A} f_{h}\left(X u_{h}\right) d x+\int_{A^{\prime}}\left(f_{h}\left(X\left(\rho_{\epsilon} * \bar{u}_{h}\right)\right)-f_{h}\left(\rho_{\epsilon} * \overline{X u_{h}}\right)\right) d x \\
& =F_{h}^{*}\left(u_{h}, A\right)+R_{\epsilon, h} .
\end{aligned}
$$

From (101), (112) and (113), it follows that, for given $0<\varepsilon<\operatorname{dist}\left(A^{\prime}, \mathbb{R}^{n} \backslash\right.$ A)

$$
\lim _{h \rightarrow \infty} R_{\epsilon, h}=R_{\epsilon}:=\int_{A^{\prime}}\left(f\left(X\left(\rho_{\epsilon} * \bar{u}\right)\right)-f\left(\rho_{\epsilon} * \overline{X u}\right)\right) d x .
$$


For given $0<\varepsilon<\operatorname{dist}\left(A^{\prime}, \mathbb{R}^{n} \backslash A\right.$ ), by (107), (109), (114), and (116), passing to the limit in (115) as $h \rightarrow \infty$, it follows that

$$
\begin{aligned}
\tilde{F}\left(\rho_{\varepsilon} * \bar{u}, A^{\prime}\right) & \leq \liminf _{h \rightarrow \infty} F_{h}^{*}\left(\rho_{\varepsilon} * \bar{u}_{h}, A^{\prime}\right) \\
& \leq \lim _{h \rightarrow \infty} F_{h}^{*}\left(u_{h}, A\right)+\lim _{h \rightarrow \infty} R_{\epsilon, h}=F(u, A)+R_{\epsilon} .
\end{aligned}
$$

Let us now show that

$$
\lim _{\epsilon \rightarrow 0^{+}} R_{\epsilon}=0
$$

Indeed

$$
X\left(\rho_{\epsilon} * \bar{u}\right) \rightarrow X u \text { and } \rho_{\epsilon} * \overline{X u} \rightarrow X u \text { in } L^{p}\left(A^{\prime}\right) \text {, as } \epsilon \rightarrow 0^{+}
$$

and

$f\left(X\left(\rho_{\epsilon} * \bar{u}\right)\right) \leq c_{1}\left(1+\left|X\left(\rho_{\epsilon} * \bar{u}\right)\right|^{p}\right)$ and $f\left(\rho_{\epsilon} * \overline{X u}\right) \leq c_{1}\left(1+\left|\rho_{\epsilon} * \overline{X u}\right|^{p}\right)$ a.e. in $A^{\prime}$.

Since $f$ is continuous, from Vitali's convergence theorem, (118) follows. By the semicontinuity of $\tilde{F}$, with respect to the $L^{p}$-topology, and by (118), we can pass to the limit as $\varepsilon \rightarrow 0^{+}$in (117) and we get

$$
\tilde{F}\left(u, A^{\prime}\right) \leq \lim _{\varepsilon \rightarrow 0^{+}} \tilde{F}\left(\rho_{\varepsilon} * \bar{u}, A^{\prime}\right) \leq F(u, A) \text { for each } A^{\prime} \Subset A .
$$

Finally, taking the supremum in (119) on all $A^{\prime} \in \mathcal{A}$ with $A^{\prime} \Subset A$, we get (106).

\section{REFERENCES}

[ACM] F. ACANFora, G. CARdone, S. Mortola, On the variational convergence of nonequicoercive quadratic integral functionals and semicontinuity problems, NoDEA 10 (2003), 347-373.

[APS] L. Ambrosio, A. Pinamonti, G. Speight, Weighted Sobolev Spaces on Metric Measure Spaces, Journal fur die Reine und Angewandte Mathematik (Crelle's Journal) 746 (2019), 39-65.

[BFTT] A. Baldi, B. Franchi, N. Tchou, M.C. Tesi, Compensated compactness for differential forms in Carnot groups and applications, Adv. Math. 223 (2010), 15551607.

[BFT] A. Baldi, B. Franchi, M.C. Tesi, Compensated compactness, div-curl theorem and H-convergence in general Heisenberg groups, Lect. Notes Seminario Interdisciplinare di Matematiche 6 (2007), 33-47.

[BMT] M. Biroli, U. Mosco, N. TCHOU, Homogenization for degenerate operators with periodic coefficients with respect to the Heisenberg group, C. R. Acad. Sci. Paris, $\mathbf{3 2 2}$ (1996)., Srie I 439-444.

[BPT1] M. Biroli, C. PicARD, N. TChou, Homogenization of the p-Laplacian associated with the Heisenberg group, Rend. Accad. Naz. Sci. XL Mem. Mat. Appl. (5) 22 (1998), 23-42.

[BPT2] M. Biroli, C. Picard, N. TCHOU, Asymptotic behavior of some nonlinear subelliptic relaxed Dirichlet problems Rend. Accad. Naz. Sci. XL Mem. Mat. Appl. (5) 26 (2002), 55-113.

[BT] M. BIROLI, N. TCHOU, Г-convergence for strongly local Dirichlet forms in perforated domains with homogeneous Neumann boundary conditions, Adv. Math. Sci. Appl. 17 (2007), no. 1, 149-179. 
[BLU] A. Bonfiglioli, E. Lanconelli, F. Uguzzoni, Stratified Lie Groups and Potential Theory for Their Sub-Laplacians, Springer, 2007.

[Bra] A. BRAIDEs, A handbook of $\Gamma$-convergence, Handbook of Differential Equations.Stationary Partial Differential Equations 3, M. Chipot and P. Quittner, eds., Elsevier, 2006.

[B] G. Buttazzo, Semicontinuity, relaxation and integral rapresentation, Longman, Harlow, 1989.

[DM] G. Dal Maso, An Introduction to $\Gamma$-convergence, Birhkäuser, Boston, 1993.

[ET] I. Ekeland, R. TÉman, Convex Analysis and Variational Problems, Classic in Applied Mathematics 28, SIAM , Philadelphia, 1999.

[FS] G.B. Folland, E.M. Stein, Hardy spaces on homogeneous groups, Princeton University Press, Princeton, 1982.

[FGVN] B. Franchi, C.E. Guitiérrez, T. van Nguyen, Homogeneization and Convergence of Correctors in Carnot Groups, Comm. PDE 30 (2005), 1817-1841.

[FSSC1] B. Franchi, R. Serapioni, F. Serra Cassano, Meyers-Serrin type theorems and relaxation of variational integrals depending on vector fields, Houston J. Math., 22 (1996), 859-889.

[FSSC2] B. Franchi, R. Serapioni, F. Serra Cassano, Approximation and Imbedding Theorems for Weighted Sobolev Spaces Associated with Lipschitz Continuous Vector Fields, Bolletino U.M.I, 11-B (1997), 83-117.

[FTT] B. Franchi, N. Tchou, M.C. Tesi, Div-Curl Type Theorem, H-convergence and Stokes Formula in the Heisenberg Group, Comm. Contemp. Math. 81, no. 1 (2006), 67-99.

[FT] B. Franchi, M.C. Tesi, Two-scale convergence in the Heisenberg group, J. Math. Pures Appl. 81 (2002), 495-532.

[Fu] M. Fukushima, Dirichlet Forms and Symmetric Markov Processes, North-Holland, Amsterdam, 1980.

[GN] N.Garofalo, D.M Nhieu, Isoperimetric and Sobolev Inequalities for CarnotCarathéodory spaces and the existence of minimal surfaces, Comm. Pure Appl. Math., 49 (1996), 1081-1144.

[La] P. D. LAx, Linear Algebra and its Applications- 2nd Ed., Wiley, Hoboken, NewJersey, 2007.

[MR] Z.-M. MA, M. RöcKNER, Introduction to the Theory of (Non-symmetric) Dirichlet forms, Universitext, Springer-Verlag Berlin Heidelberg 1992.

[MPSC] A. Maione, A. Pinamonti, F. Serra Cassano, $\Gamma$-convergence for functionals depending on vector fields. II. Convergence of minimizers., forthcoming.

[MS] N.G. Meyers, J. Serrin, $H=W$, Proc. Nat. Acad. Sci. USA, 51 (1964), 10551056 .

[Mo] U. Mosco, Composite Media and Asymptotic Dirichlet Forms, J. Funct. Anal. , 123 (1993) , 368-421.

[Ro] R.T. Rockafellar, Convex Analysis, Princeton University Press, Princeton, 1972.

Alberto Maione: Dipartimento di Matematica, Università di Trento, Via Sommarive 14, 38123, Povo (Trento) - Italy,

E-mail address: alberto.maione@unitn.it

Andrea Pinamonti: Dipartimento di Matematica, Università di Trento, Via Sommarive 14, 38123, Povo (Trento) - Italy,

E-mail address: andrea.pinamonti@unitn.it

Francesco Serra Cassano: Dipartimento di Matematica, Università di Trento, Via Sommarive 14, 38123, Povo (Trento) - Italy,

E-mail address: cassano@science.unitn.it 